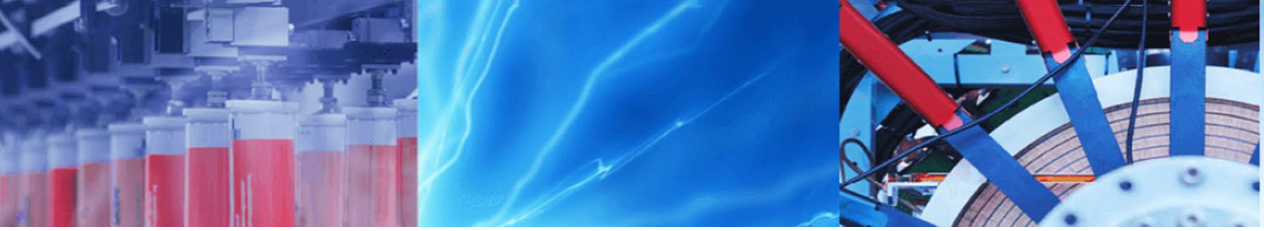

Research Article

\title{
A 3D CFD comparative study between torsioned and non-torsioned net-type feed spacer in reverse osmosis
}

\author{
Mohamed Medhat A. Abdelbaky ${ }^{1}$. Mohamed M. El-Refaee $^{1}$
}

(c) Springer Nature Switzerland AG 2019

\begin{abstract}
Spacer-filled channels are incorporated in membrane modules in reverse osmosis applications where the role of feed spacer is to promote turbulence, improve mass transport and mitigate concentration polarization. The objective of this study is to emulate a realistic spacer, due to manufacturing conditions, spacers tend to have a special complex geometry (deformations) which is usually not adopted in computational fluid dynamics (CFD) studies, this deformation tends to be of a torsioned shape. A total of 18 simulations were conducted for torsioned and non-torsioned spacers evaluating concentration polarization and mass transfer across membrane walls at different inlet salinities $\left(c_{0}=400,500\right.$ and $\left.600 \mathrm{~mol} / \mathrm{m}^{3}\right)$ and different Reynolds number $(\mathrm{Re}=100,192$ and 384) based on the inlet velocity using fully-coupled three dimensional CFD, where the solution-diffusion model was incorporated for the calculations of water and solute fluxes across reverse osmosis membrane. The current comparison illustrates that the torsioned spacers have an overall enhanced performance compared to non-torsioned spacers. It is found that concentration polarization is mitigated in torsioned spacers which is the contrary with non-torsioned spacers having a tendency to concentration polarization, consequently, the water flux in torsioned spacers was found higher than those in non-torsioned spacers. Generally speaking, all membrane modules are found to have a higher performance at higher Reynolds number and at lower inlet salinity. This study proves that spacer geometric details are critical to predict accurately the RO membrane performance.
\end{abstract}

Keywords Reverse osmosis (RO) · Computational fluid dynamics (CFD) · Feed channel · Concentration polarization · Mass transfer

\section{List of symbols}

A Water permeability $[\mathrm{m} /(\mathrm{Pa} \mathrm{s})]$

B Solute permeability $\left[\mathrm{mol} /\left(\mathrm{m}^{2} \mathrm{~s}\right)\right]$

c Concentration $\left(\mathrm{mol} / \mathrm{m}^{3}\right)$

$d_{h} \quad$ Hydraulic diameter $(\mathrm{m})$

$D_{s} \quad$ Diffusion coefficient $\left(\mathrm{m}^{2} / \mathrm{s}\right)$

$J_{w} \quad$ Water flux $\left(\mathrm{m}^{3} / \mathrm{m}^{2} \mathrm{~s}\right)$

$J_{s} \quad$ Solute flux $\left(\mathrm{mol} / \mathrm{m}^{2} \mathrm{~s}\right)$

$k \quad$ Osmotic factor $\left(\mathrm{m}^{3} \mathrm{~Pa} / \mathrm{mol}\right)$

$n$ Normal vector (dimensionless)

$p \quad$ Pressure $(\mathrm{Pa})$

$R \quad$ Gas constant $[\mathrm{J} /(\mathrm{mol} \mathrm{K})]$

$T$ Temperature (K)

u Velocity vector $(\mathrm{m} / \mathrm{s})$ $x \quad$ Cartesian coordinate

y Cartesian coordinate

$z \quad$ Cartesian coordinate

Greek letters

$\rho \quad$ Density $\left(\mathrm{kg} / \mathrm{m}^{3}\right)$

$\mu \quad$ Dynamic viscosity (Pa s)

$\pi \quad$ Osmotic pressure $(\mathrm{Pa})$

\begin{tabular}{ll}
\multicolumn{2}{l}{ Subscripts } \\
ave & Average \\
b & Bulk \\
f & Feed \\
low & Lower membrane surface \\
o & Inlet condition
\end{tabular}

Mohamed Medhat A. Abdelbaky, mohamed.medhat@must.edu.eg; Mohamed M. El-Refaee, mohamed.elrefaee@must.edu.eg | ${ }^{1}$ Department of Mechanical Engineering, Misr University for Science and Technology, Giza, Egypt.

SN Applied Sciences (2019) 1:1059| https://doi.org/10.1007/s42452-019-1098-8 
p Permeate

up Upper membrane surface

Abbreviations
CFD Computational fluid dynamics
CP $\quad$ Concentration polarization
Re $\quad$ Reynolds number
RO

\section{Introduction}

Reverse osmosis is a desalination technique widely used around the world to address the problem of water scarcity and overcome the fresh water shortages. The membrane in spiral wound membrane, is semi-permeable characterized by passing the pure water and blocking the salt ions from passing. Some researches assume complete salt rejection and no solute flux is present, and others assume constant solute flux [1-3]. In order to model the reverse osmosis across membrane properly and predict membrane performance accurately, boundary conditions should emulate realistic $\mathrm{RO}$ membranes regarding the water and solute fluxes, consequently the membrane is treated as a functional surface where the mass transport is obtained by the local pressure and local concentration, and constant solute and water flux are avoided. As a result of salt rejection, the phenomena of salt accumulation on membrane walls is denoted as concentration polarization. This phenomenon affects the membrane performance in a way that increases the osmotic pressure across the membrane which leads to a degraded membrane performance.

Three-dimensional fluid dynamics simulation requires huge amounts of computational resources, and simulation time can be extremely lengthy [4] which sometimes leads to oversimplified models, and oversimplified models leads to inaccurate results and inefficient RO element performance estimation [5]. However, Computational fluid dynamics (CFD) — within the past 10 years - has reached the level of sophistication required to effectively describe the flow around spacer filaments and to investigating mass transfer behavior [4]. Karode and Kumar [6] are considered the first to conduct a three dimensional (3D) hydrodynamics simulation in spacer filled channels using several commercially available spacer configurations. They analyzed the performance of each spacer configuration based on the degree of bulk fluid mixing, drag coefficient and average shear rate at Reynolds number (225-2225) based on the channel height and the bulk velocity. They reported that the fluid flows parallel to the feed spacer not in zigzag as was suggested before in previous released researches. Additionally, higher pressure drop occurred in spacers with equal filament diameters, and more uniform shear rate was observed at the upper and lower surfaces of the tested cells.

Cao et al. [5] modeled a feed channel containing 2 spacers filaments using the turbulent RNG k-e model to obtain time-average velocity profile. They found that the improvement of mass transport through the walls is directly associated with; the high shear stress, velocity fluctuation and eddy formation, also they found that reducing the distance of transverse filament leads to the enhancement of mass transport due to larger shear stress regions near the walls. Koutsou et al. [7] performed a 3D direct numerical simulation (DNS) using periodic boundary conditions to study the hydrodynamics of the feed flow in spacer filled channel with impermeable membrane in the cases of steady and unsteady flow regimes. They conducted their simulations with Reynolds number 35 and 300 (on the basis of cylinder diameter and bulk velocity), although they did not consider mass transfer, they concluded that the flow in spacer-filled channels becomes unsteady with Reynolds number ranged between 35 and 45 . Fimbres-Weis and Wiley [8] used steady 3D flow and mass transfer simulation in narrow non-woven spacer-filled channels (angles $45^{\circ}$ and $90^{\circ}$ ) for Schmidt number 600 and Reynolds number up to 200. They assumed the membrane to be impermeable, and the mass transfer coefficient was calculated from an empirical formula stating that the mass transfer and pressure drop are influenced by the spacer configurations and by feed flow rate. Ranade and Kumar [9] performed a periodic unit cell to simulate rectangular and curvilinear spacer filled channels for the first time, which could give us a useful understanding of the fluid behavior in spacer filled modules. They have found that there is not a considerable difference between the results obtained from circular and flat channels, accordingly flat channels can be used in studying SWM modules.

Srivathsan [10] have concluded 3D computational fluid dynamics to investigate the membrane performance, the study was performed on a computational cell containing two crossing spacers using periodic conditions in the streamwise direction. Simulations were done by assuming the membrane to be permeable and in another case to be impermeable, however in both cases Sherwood number was calculated using an empirical relation and it was confirmed that the membrane performance and the pressure drop were highly influenced by the spacing between spacers. Boram et al. [11] conducted a 3D CFD simulation on different types of feed spacers configurations with different geometrical variations. The solution diffusion model was implemented in the simulation for the calculations of solute and water fluxes across the membrane. They have found that the fully woven spacers have a higher tendency in mitigating concentration polarization and delivering the highest water flux when compared with other spacer 
configurations. Anqi et al. [12] studied a 3D steady multicomponent fluid flow in spacer filled channels using the SST $k-\omega$ turbulence model evaluating the hydrodynamics and concentration polarization on the membrane walls with different spacers configurations at $\operatorname{Re}=400$ and 800 . They found generally that all membranes have a higher performance at higher flow rates and that the most efficient one was achieved by the $30^{\circ}$ spacers arrangement indicating the crucial importance of feed spacers configuration for the enhancement of $\mathrm{RO}$ modules.

From this review, it is clearly found that the literature lacks the investigation of 3D models of non-woven nettype spacers with realistic spacer geometries to study the flow and concentration polarization of a non-constant spacer diameter as spacers tend to have a complex geometry (deformation) due to manufacturing conditions. The spacer with this type of deformation is denoted in the current study as torsioned spacer. A steady three-dimensional computational study is performed to evaluate the velocity fields, concentration polarization, water and solute fluxes, and the pressure drop across the membrane at different Reynolds number and at different inlet salinity in cases of torsioned and non-torsioned feed spacers. The comparison between the two aforementioned spacers should give us an insight of how critical and important is the geometric details to predict accurately the performance of membranes in RO.

The current author used the solution-diffusion model which is widely used in simulating mass transport across RO membranes [10-13], the mentioned model includes a relation between the solute flux, the solute permeability and the concentration difference across the membrane, consequently, the solute concentration in the permeate side can be predicted in conjunction with the calculated solute fluxes [11].

\section{Mathematical model and mesh independency}

A unit cell is incorporated for all simulations containing torsioned and non-torsioned spacers, as it was proved in the literature, the fully developed fields are achieved after just a few unit cells [14]. It is presented in Fig. 1 the detailed schematics of the unit cell used in the computational domain. It is important to mention that the geometric schematics for torsioned and non-torsioned spacers are brought from an experimental study shown in Fig. 2, performed by Haidari et al. [15] which are the results of an average of at least ten measurements in the laboratory of a non-woven commercial feed spacer manufactured by Delstar Technologies. Accordingly, feed spacer height is chosen to be $0.86 \mathrm{~mm}$ and the maximum spacer diameters for torsioned and non-torsioned spacers are $0.49 \mathrm{~mm}$. Also, the flow attack angle is equal to $45^{\circ}$ and the hydrodynamic angle to be $90^{\circ}$. As illustrated in Fig. $1 \mathrm{~b}$, the torsioned spacer is designed with 7 different diameters following the same ratio of cross-section reduction found in Fig. 2, in such way, simulating a realistic feed spacer like the ones found commercially.

The inlet feed stream flows perpendicular to the inlet and outlet faces along the $\mathrm{x}$-axis, and permeations are taking place on the upper and lower faces of the unit cell in z-direction representing the membrane surfaces. Furthermore, the concentration layers on the membrane walls are calculated by the upcoming presented models avoiding constant concentration values. The flow and mass transfer inside the reverse osmosis membrane channels can be described by coupling the continuity equations for the flow (Eqs. 1 and 2), and the convection-diffusion equation for the mass transport (Eq. 3), assuming the fluid used to be incompressible and Newtonian as follows;

$\nabla \cdot \boldsymbol{u}=0$

$\rho \boldsymbol{u} \cdot \nabla \mathbf{u}=-\nabla p+\mu \nabla^{2} \boldsymbol{u}$

$\nabla \cdot\left(D_{s} \nabla \mathbf{u}\right)-\nabla \cdot(\boldsymbol{u c})=0$

where $u$ is the fluid velocity, $\rho$ density of fluid, $\mu$ dynamic viscosity, $p$ pressure, $c$ solute concentration and $D_{s}$ the diffusion coefficient.

Each unit cell includes 4 types of boundaries: the inlet, outlet, spacer walls and membrane surfaces. The boundary conditions for the inlet were set as follows: inlet velocity $\left(u_{o}\right)=0.052,0.1$ and $0.2(\mathrm{~m} / \mathrm{s})$ corresponding to $R e=100$, 192 and 384.5 respectively. Also, the inlet concentration was set to $\left(c_{o}\right)=400,500$ and $600\left(\mathrm{~mol} / \mathrm{m}^{3}\right)$ having a total number of 18 simulations for torsioned and non-torsioned spacers.

The set of equations and boundary conditions adopted in this simulation are the same as the one used by Boram et al. [11] where constant pressure is applied at the outlet bound$\operatorname{ary}(p)=60 \times 10^{5}(\mathrm{~Pa})$, and zero solute flux with no slip condition is used along the surfaces of the filament walls. The side faces are treated as periodic boundary conditions for flow and solute transport. The convection-diffusion equation used in this study assumes the RO membrane as a functional surface where the water velocity is calculated from the trans-membrane pressure difference and the osmotic pressure across the membrane as follows;

$J_{w}=A \cdot\left(\Delta P_{\text {memb }}-\Delta \pi_{\text {memb }}\right)$

$\Delta P_{\text {memb }}=P_{f}-P_{p}$

$\Delta \pi_{m e m b}=\pi_{f}-\pi_{p}$

$\Delta \pi_{\text {memb }}=k\left(c_{f}-c_{p}\right)$ 


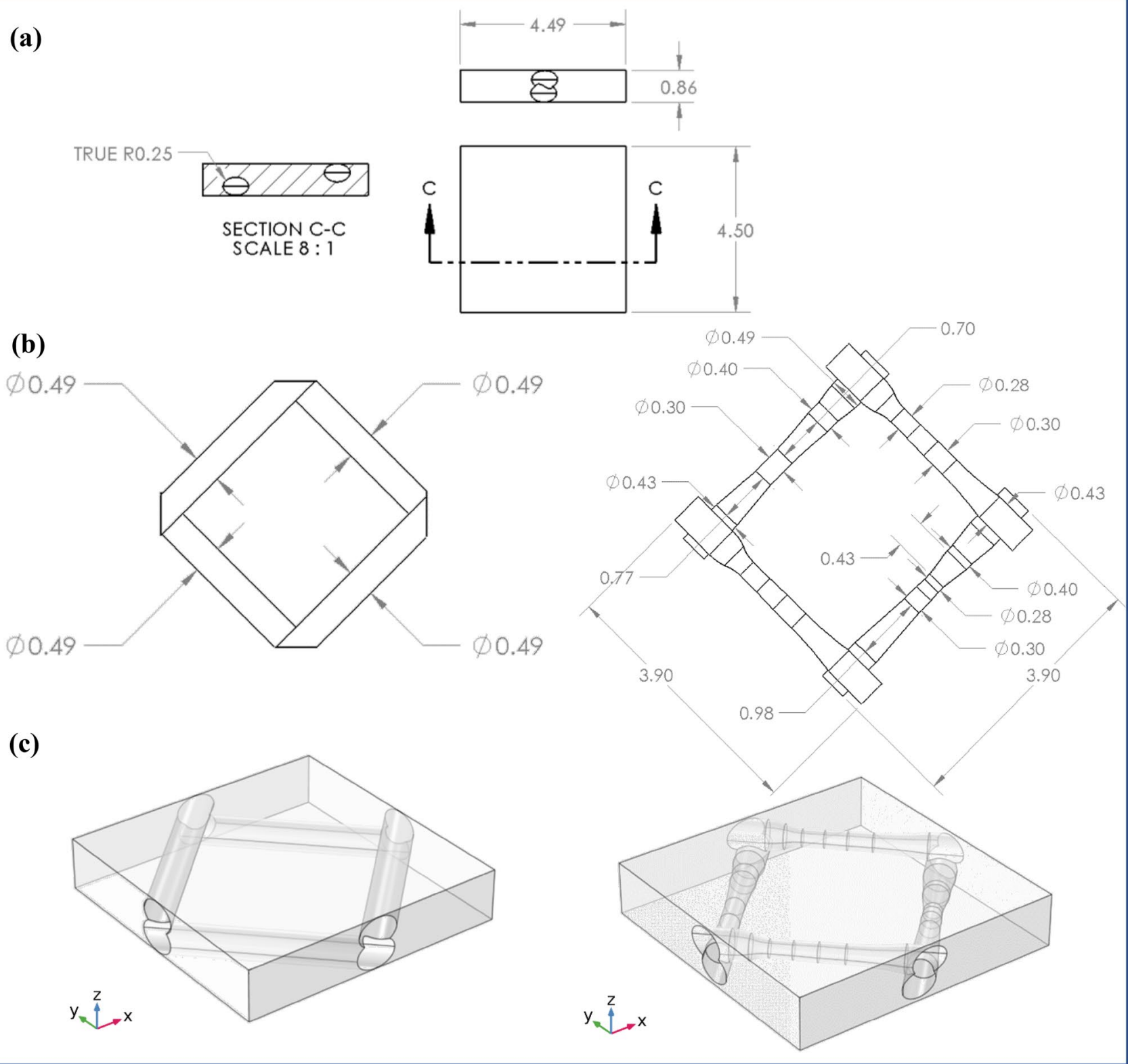

Fig. 1 Schematics of the investigated unit cell a dimensions of the unit cell $\mathbf{b}$ dimensions of non-torsioned and torsioned spacers $\mathbf{c}$ isometric view of the unit cell

Fig. 2 The typical commercial non-woven feed spacer a filament dimensions and $\mathbf{b}$ distance between the spacer and the membrane walls [20]
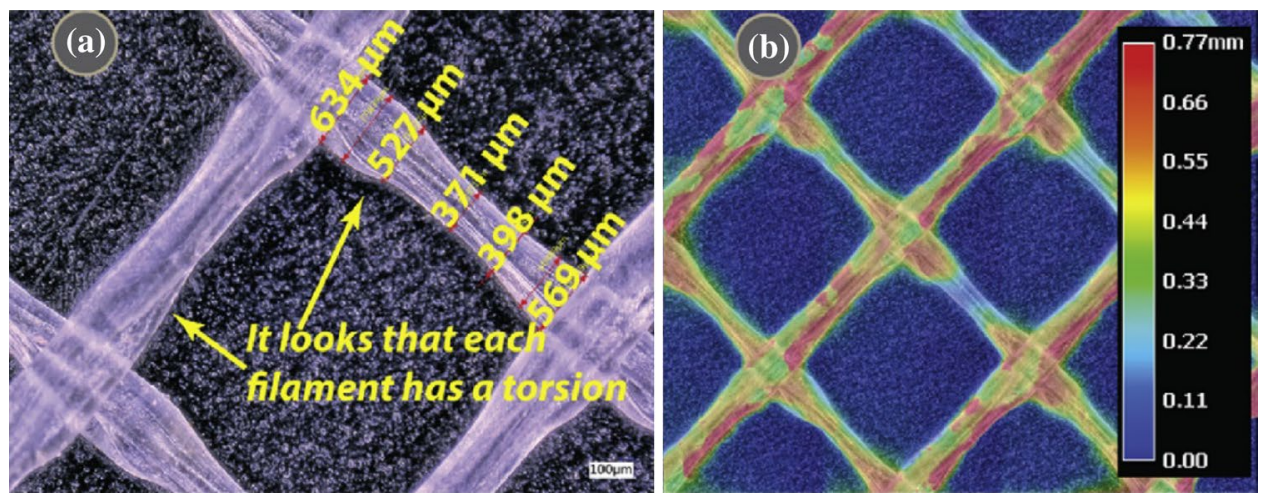
where $J_{w}$ is the water flux, $A$ the membrane permeability, $\Delta P_{\text {memb }}$ static pressure difference across the membrane, $\Delta \pi_{\text {memb }}$ is the osmotic pressure difference across the membrane and the difference between $\Delta P_{\text {memb }}$ and $\Delta \pi_{\text {memb }}$ is called the net difference pressure NDP which is responsible of driving the flow through the membrane. The NDP should be positive for the water to flow from the feed side to the permeate side. In other words, the osmotic pressure drives the flow from low concentrate region to a higher concentrate region [10]. RO membranes have the ability to remove multi-solute contaminants, but for simplicity, a single solute will be adopted in this research. Water will be the solvent and $\mathrm{NaCl}$ would be the solute, thus the salt flux $J_{s}$ is presented as follows;

$J_{s}=B\left(c_{f}-c_{p}\right)$

where $J_{s}$ is the solute flux, $B$ as the salt permeability, $C_{f}$ feed concentration and $c_{p}$ as the permeate concentration. Concerning the calculation of the permeate concentration $c_{p}$, it is possible to re-write (Eqs. 4-8) as the feed pressure and concentration are calculated from (Eqs. 1-3) by combining them to be as follows;

$k A c_{p}^{2}+\left[A\left(\Delta P_{\text {memb }}-k c_{f}\right)+B\right] c_{p}-B c=0$

Despite its value to be higher than the atmospheric pressure in a small extent, the permeate pressure is assumed to be zero [11]. The $c_{p}$ is written from (Eq. 9) to be as follows;

$c_{p}=\frac{-X_{1}+\sqrt{X_{1}^{2}+X_{2}}}{2 k A}$

where $X_{1}=A\left(P_{f}-k c_{f}\right)+B$ and $X_{2}=4 k A B c_{f}$.

The water and solute fluxes can be calculated using (Eqs. 4 and 8) after obtaining the $c_{p}$ from (Eq. 10). As it is assumed that the water flux flows perpendicularly to the membrane walls, the water velocity at the membrane walls can be presented as follows [11];

$u_{u p}=\left[\begin{array}{lll}0 & 0 & J_{w}\end{array}\right]$

$u_{\text {low }}=\left[\begin{array}{lll}0 & 0 & -J_{w}\end{array}\right]$

where $u_{l o w}$ and $u_{u p}$ are the water velocity at the lower and upper membrane surfaces, respectively. As presented in (Eqs. 11 and 12) There is no water flowing in the $x$ and $y$ directions, and the only water flow occurs at the z-direction, and the negative sign denotes that the water flux leaves the domain. Solute fluxes across the membrane surfaces can be illustrated as follows;

$-\boldsymbol{n} .\left(D_{s} \nabla c-u c\right)=-J_{s}$
Membrane permeability is selected from the literature to be $A=2.5 \times 10^{-12}[\mathrm{~m} /(\mathrm{s} \mathrm{Pa})]$, salt permeability $B=2.5 \times 10^{-8}(\mathrm{~m} / \mathrm{s})$, osmotic factor $k=4958\left(\mathrm{~m}^{3} \mathrm{~Pa} / \mathrm{mol}\right)$, diffusion coefficient $D_{s}=1 \times 10^{-9}\left(\mathrm{~m}^{2} / \mathrm{s}\right)$, density $\rho=998.2$ $\left(\mathrm{kg} / \mathrm{m}^{3}\right)$, viscosity $\mu=8.93 \times 10^{-4}$ (Pa s) and temperature $T=25^{\circ} \mathrm{C}[11]$.

All the models included in this study were designed in Solidworks 2017, meshed in ANSYS 19.0 then imported into Comsol Multiphysics 5.4 to be solved. Comsol Multiphysics uses the Galerkin finite element method which converts differential equations in a continuous domain into a discrete problem to solve governing equations over a computational mesh [13]. The mesh is constructed of triangular elements located through the domain with thin quadrilateral elements at the boundaries (Fig. 3). It had also to be ensured that the mesh is optimized in a way that isn't exaggerated in means of computational resources and at the same time doesn't produce significant artifacts, accordingly a mesh dependency was performed and the shear rate on the upper membrane was calculated, as can be seen in Fig. 4, no significant differences occurred between the different meshes. The final mesh chosen in simulations are 0.4 and 0.5 million elements for the nontorsioned and torsioned spacers, respectively, varying on the basis of the spacer design and the volume of the fluid domain. The simulations were conducted in two stages where in the first stage, no flux is assumed at the membrane surfaces, then the solution of the first stage is used as an initial guess for the second stage using the boundary conditions at the membrane surfaces illustrated earlier. MUMPS - which is a parallel sparse direct solver-is employed to solve the coupled fluid and mass transport equations, all simulations are converged within 25 iterations with a convergence criterion of $10^{-4}$ using AWS Amazon \& Google Cloud Platform with a 32 vCPU (16 cores) and a $150 \mathrm{~GB}$ of RAM.

\section{Results and discussion}

First of all, the calculated velocity and concentration profiles from the unit cell are demonstrated so the difference between the non-torsioned and torsioned spacers can be observed. Secondly, the water and solute flux are presented to evaluate the membrane performance in both cases along with their distributions on membrane surfaces.

\subsection{Velocity contours and concentration polarization}

The results obtained from the flow simulations and concentration polarization are presented at $\operatorname{Re}=100,192.3$ 


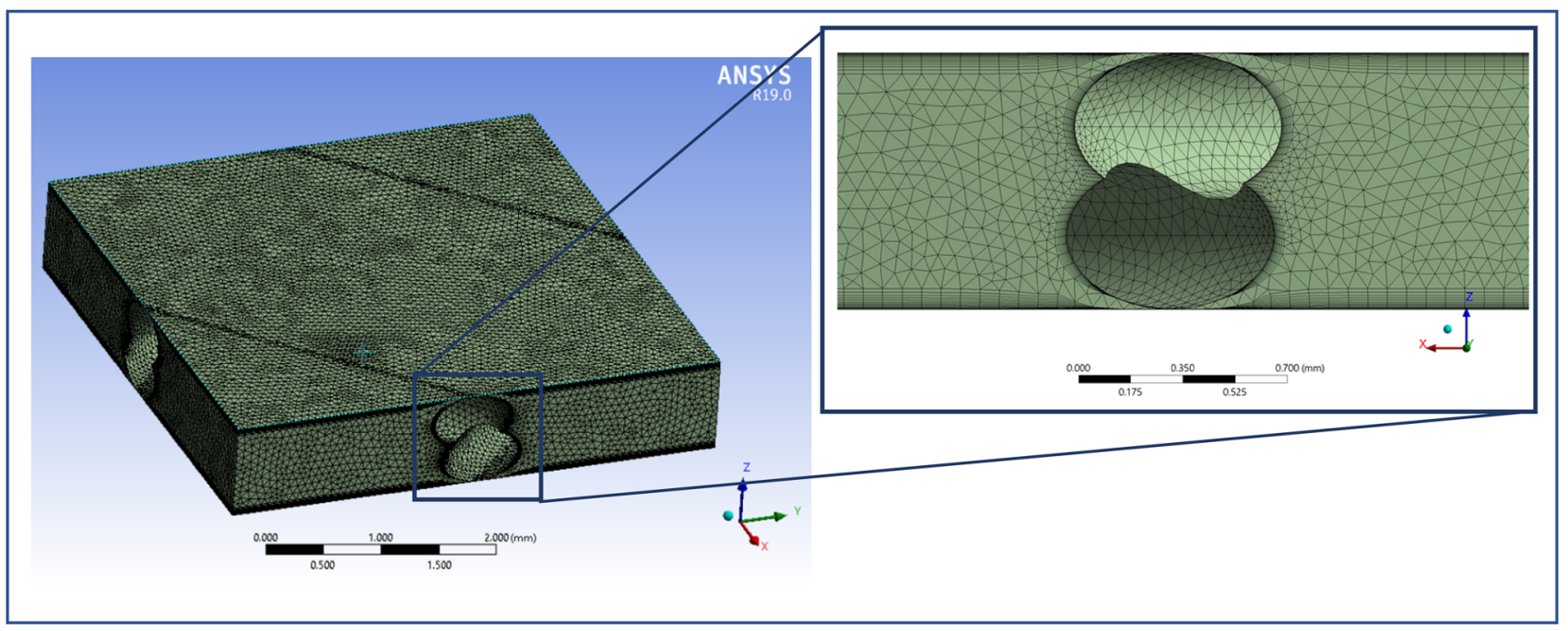

Fig. 3 Close-up view of the inflation layers used in mesh near membrane and spacer
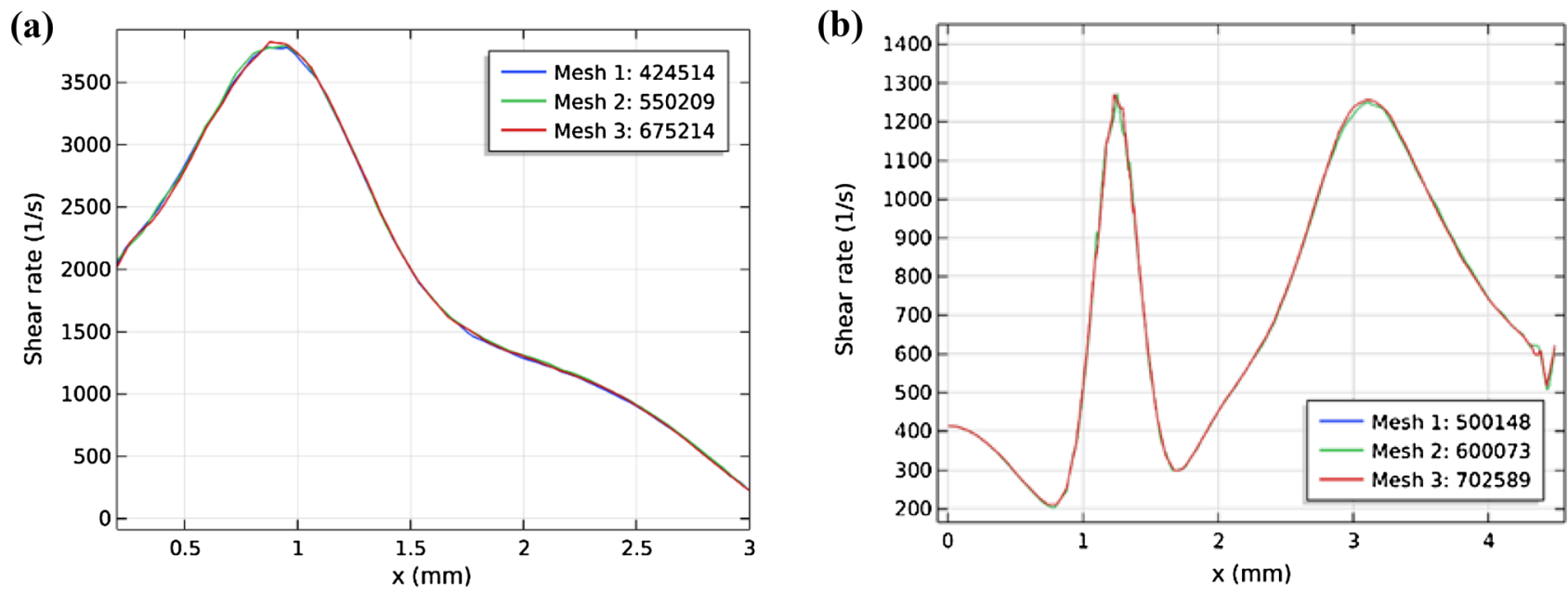

Fig. 4 Shear rates at the upper membrane calculated using 3 different meshes for $\mathbf{a}$ non-torsioned spacer and $\mathbf{b}$ torsioned spacer

and 384.5 , and at inlet salinity $\left(\mathrm{c}_{\mathrm{o}}\right)=400,500$ and $600 \mathrm{(mol} /$ $\mathrm{m}^{3}$ ) for the torsioned and non-torsioned spacers. Figures 5 and 6 illustrates the contours of velocity magnitude and streamlines comparing the non-torsioned and torsioned feed spacers at 3 different values of Reynolds number at constant inlet salinity $\left(c_{0}\right)=400\left(\mathrm{~mol} / \mathrm{m}^{3}\right)$. The velocity magnitudes are presented on a $5 \mathrm{yz}$ and $\mathrm{xz}$ slices along the unit cell which are perpendicular and parallel to the flow direction, respectively. The spacer forces the flow to make turns around resulting in low velocity regions, although their sizes vary, these recirculation regions are specifically seen in front of and behind the spacer where you could expect a degraded water flux and a promoted concentration polarization.
The velocity profiles are not affected in a significant way when comparing both spacer geometries; however, the streamline direction elaborated in Figs. 5 and 6 differ substantially for both spacers, it is clear that the streamlines are symmetrical with respect to the central xz plane in case of non-torsioned spacers which is the contrary with torsioned spacers having an asymmetrical distribution.

Figures 7, 8, 9 and 10 depict the normalized concentration polarization (CP) along the upper and lower membrane surfaces at the aforementioned Reynolds number and inlet salinity, it is important to mention that the upper membrane is only shown for the non-torsioned spacer because both the upper and lower membranes are almost identical, oppositely found with torsioned spacer where 


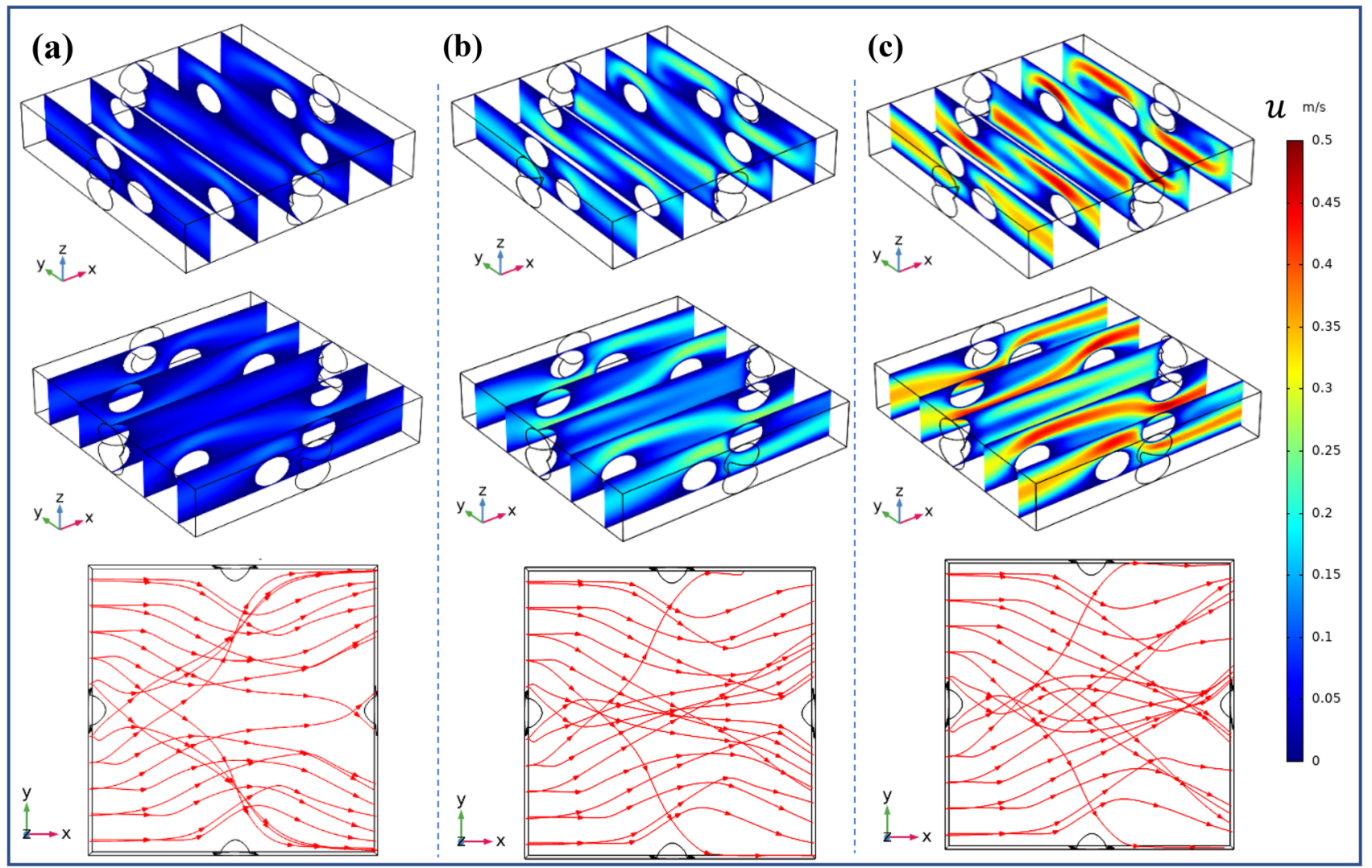

Fig. 5 Velocity profiles of non-torsioned spacers at Re $\mathbf{a} 100, \mathbf{b} 192.3$ and $\mathbf{c} 384.5$ with constant $c_{0}=400 \mathrm{~mol} / \mathrm{m}^{3}$ presented as yz and xz slices, and topview $(\mathrm{yx})$ streamline direction

membrane walls are different. At first glance, it is clear that the $\mathrm{CP}$ decreases as Reynolds number and inlet salinity increases, also the distribution of $\mathrm{CP}$ on the membrane walls are significantly different when comparing both type of spacers. In Figs. 7 and 9, it is seen that the salt accumulation is promoted above the filaments strands and at narrow regions located between the spacers and membrane walls which is due to the restriction of the solute to diffuse back to the bulk solution, mainly seen with non-torsioned spacers where the $\mathrm{CP}$ is sharply emphasized along the feed spacer. On the other hand, in torsioned spacers (Figs. 8 and $10)$, the accumulation of salt does not occur only around tight edges but also at recirculation regions around the spacers-as elaborated previously — where stagnant flow is found.

The CP magnitude in torsioned spacer is varying along its length as the spacer diameter is not constant affecting its distribution. Figure 11 is a graphical representation of the dimensionless concentration polarization $\left(c / c_{b}\right)$ for both spacers at the upper membrane for different Reynolds number and different inlet salinity. Figure 11a1, b1 illustrates the effect of increasing the inlet velocity on the concentration polarization at constant inlet salinity $\left(c_{\mathrm{o}}\right)=400\left(\mathrm{~mol} / \mathrm{m}^{3}\right)$ for non-torsioned and torsioned spacers, respectively. Similarly, Fig. 11a2, b2 shows the effect of increasing the inlet salinity at constant $R e=100$ for both spacers. It is clear from the graphs that the torsioned spacer outperform the non-torsioned spacer, for instance, the highest value reached by the torsioned spacer and non torsioned spacer are 1.07 and 2.6, respectively. The unit cell with the torsioned spacer has a wide distribution area of salt accumulation on the membrane walls, on the contrary with the non-torsioned spacer where the accumulation is seen to be formed as a spike located right above the spacers, mainly due to the disruption of the concentration boundary layer. The first and second spikes found in the torsioned spacer graphs correspond to the regions located before and after the spacer, respectively. In both cases, it is clearly seen that the concentration polarization increases as the Reynolds number and the inlet salinity decreases agreeing with the $\mathrm{CP}$ formula $c / c_{b}$. The patterns of wall concentration profiles are oppositely reflected in the water flux distribution as it is discussed in the next section. The concentrations obtained from the current study are compared with those found in the literature. Ma and Song [16] studied the effect of spacer geometry on the 


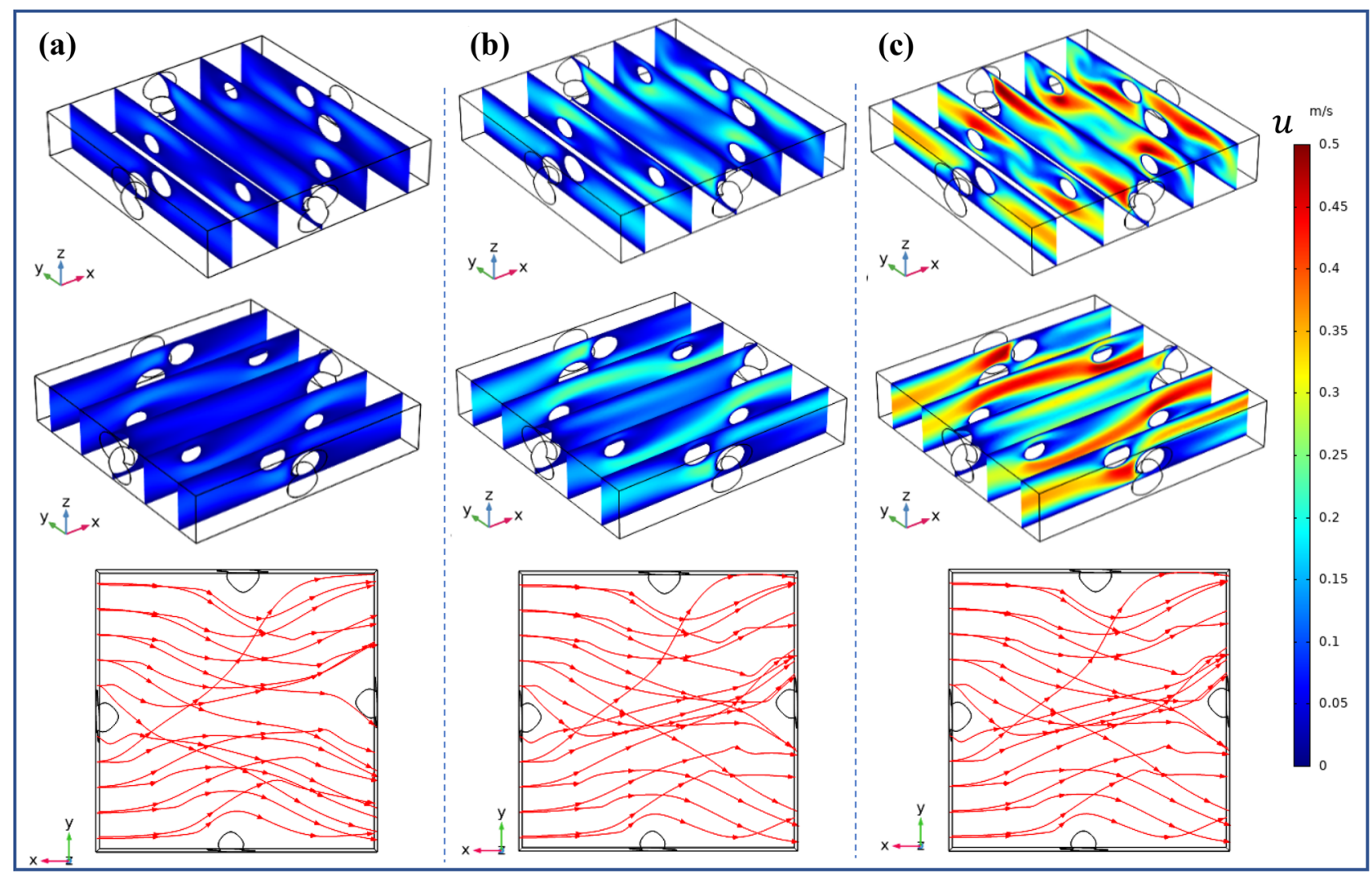

Fig. 6 Velocity profiles of torsioned spacers at Re a 100, b 192.3 and c 384.5 with constant $c_{0}=400 \mathrm{~mol} / \mathrm{m}^{3}$ presented as yz and xz slices, and topview (yx) streamline direction

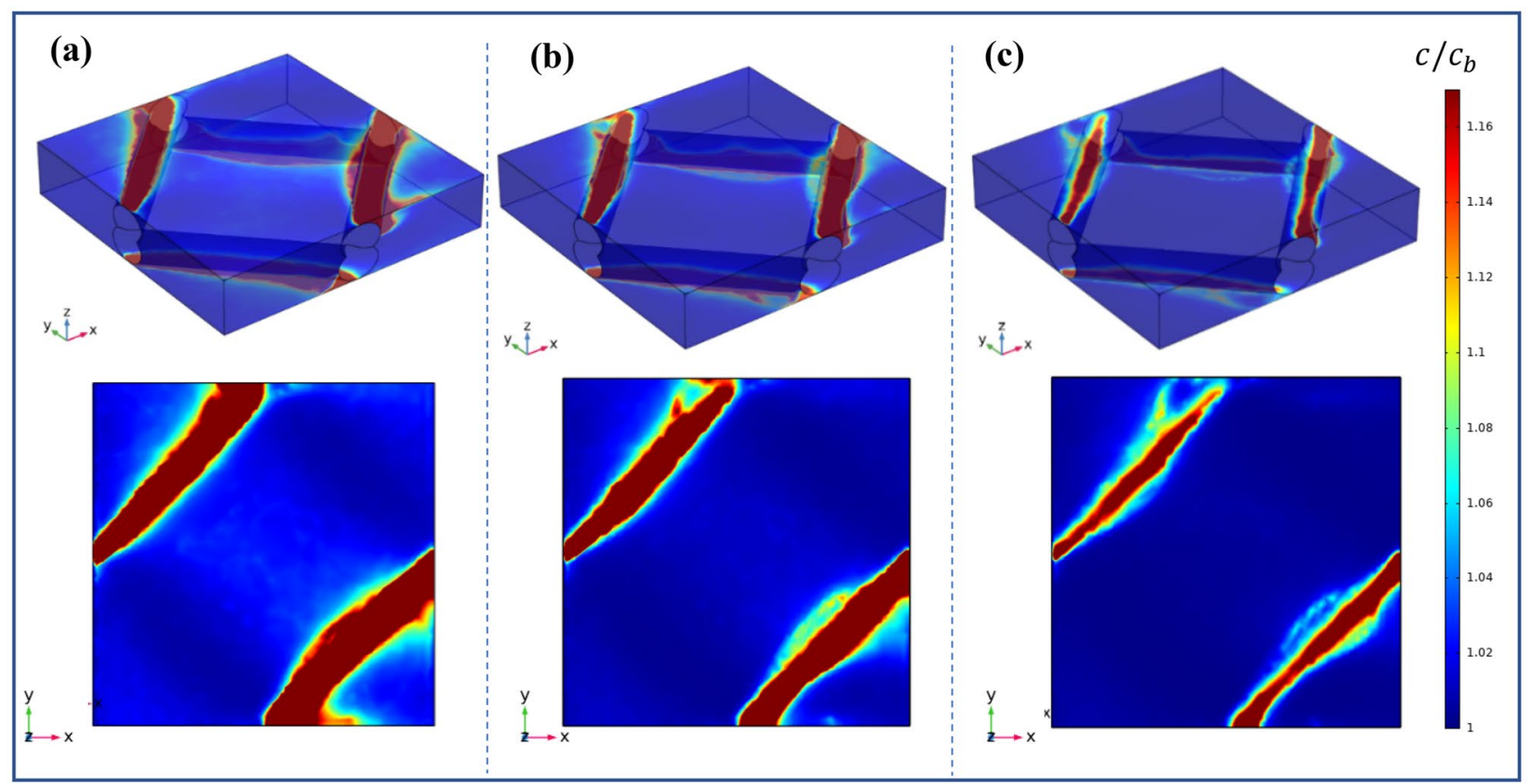

Fig. 7 Dimensionless concentration profiles $\mathrm{c} / \mathrm{c}_{\mathrm{b}}$ of non-torsioned spacers at Re a $100, \mathbf{b} 192.3$ and $\mathbf{c} 384.5$ with constant $\mathrm{c}_{\mathrm{o}}=400 \mathrm{~mol} / \mathrm{m}^{3}$ presented as isometric view and upper membrane surface 


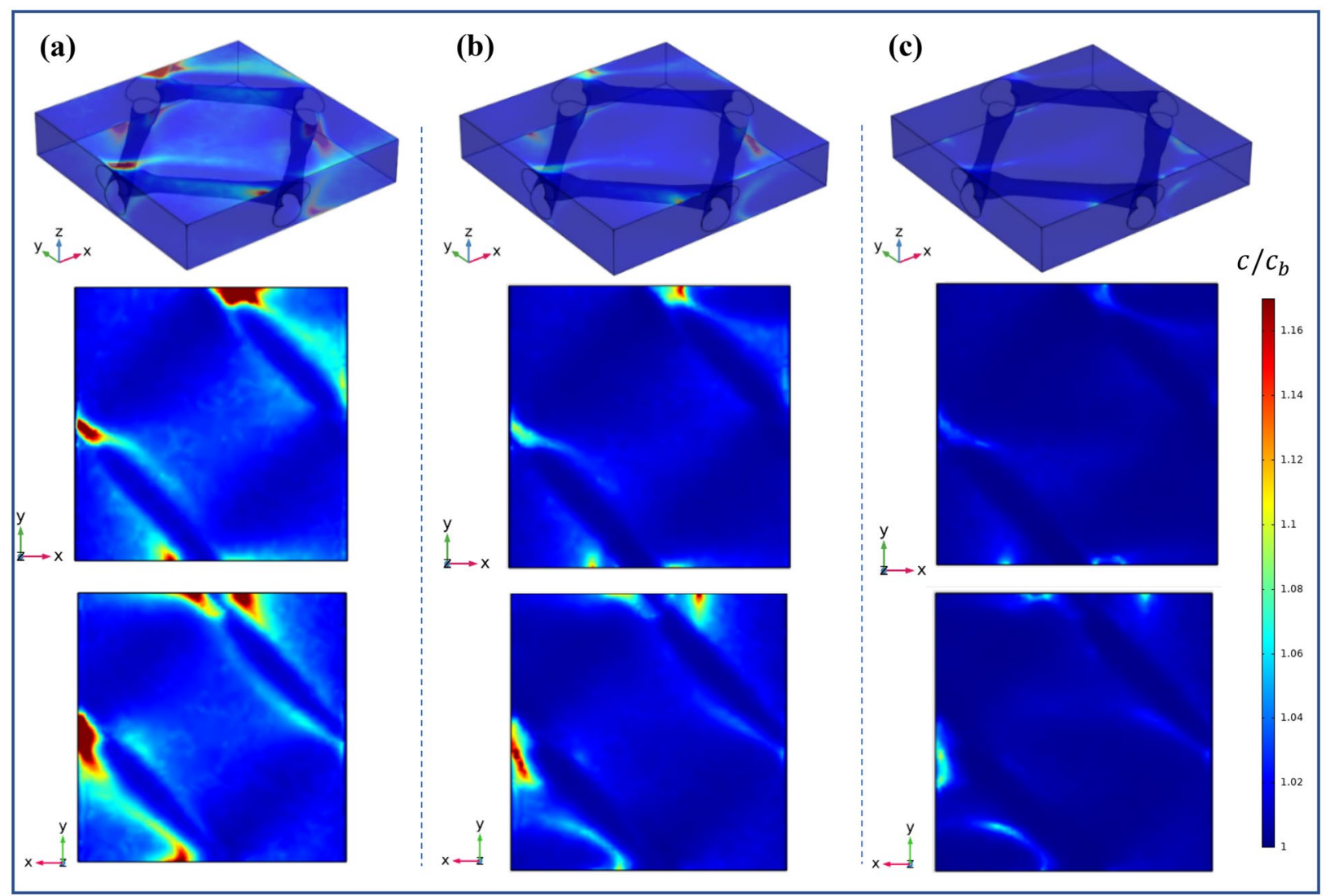

Fig. 8 Dimensionless concentration profiles $c / c_{b}$ of torsioned spacers at Re a $100, \mathbf{b} 192.3$ and c 384.5 with constant $c_{o}=400 \mathrm{~mol} / \mathrm{m}^{3} \mathrm{pre}-$ sented as isometric view and upper membrane surface and lower membrane surface

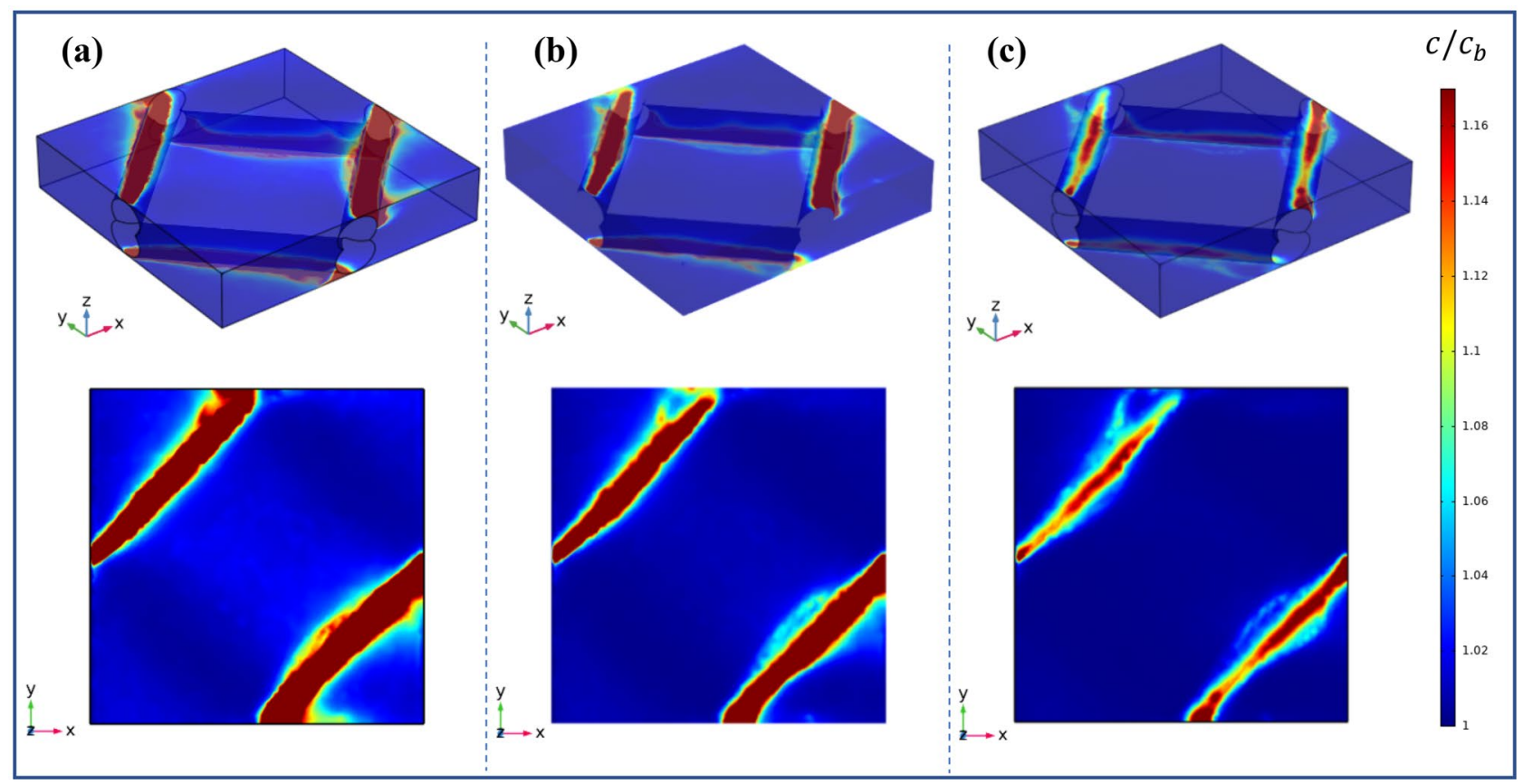

Fig. 9 Dimensionless concentration profiles $\mathrm{c} / \mathrm{c}_{\mathrm{b}}$ of non-torsioned spacers at Re $\mathbf{a} 100, \mathbf{b} 192.3$ and c 384.5 with constant $\mathrm{c}_{\mathrm{o}}=600 \mathrm{~mol} / \mathrm{m}^{3}$ presented as isometric view and upper membrane surface 


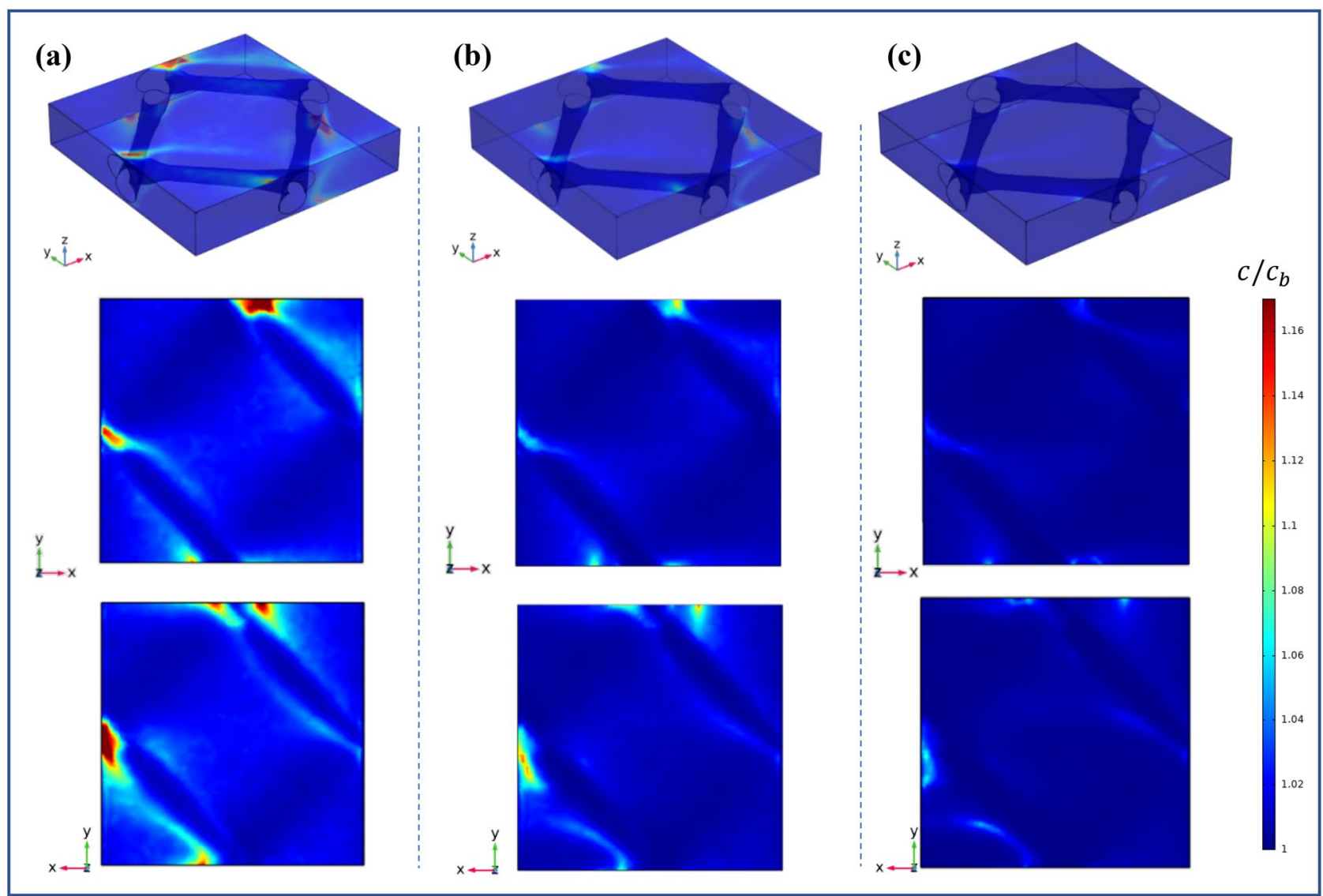

Fig. 10 Dimensionless concentration profiles $c / c_{b}$ of torsioned spacers at Re a $100, \mathbf{b} 192.3$ and c 384.5 with constant $c_{o}=600 \mathrm{~mol} / \mathrm{m}^{3}$ presented as isometric view and upper membrane surface and lower membrane surface

concentration polarization using 2D CFD study at salinity about $C_{o}=550\left(\mathrm{~mol} / \mathrm{m}^{3}\right)$ and $\mathrm{Re}=100$. They calculated the $\mathrm{CP}$ in empty feed channels to be 2.5 while those is spacer filled channels were found to be 2.10, 2.33 and 1.73 for spacer diameters of $0.5(\mathrm{~mm}), 0.25(\mathrm{~mm})$ and $0.75(\mathrm{~mm})$, respectively. Gu et al. [11] studied the impact of several spacers configurations on the CP in a 3D CFD study. They obtained the values of CP between 1.04 and 1.2 for inlet salinity $C_{o}=600\left(\mathrm{~mol} / \mathrm{m}^{3}\right)$ and $\mathrm{Re}=224$. The $\mathrm{CP}$ obtained in the current study is 2.6 for non-torsioned spacers and 1.07 for torsioned spacers at $\operatorname{Re}=100$ and $C_{o}=400\left(\mathrm{~mol} / \mathrm{m}^{3}\right)$. The CP of non-torsioned spacers agrees to a great extent with the literature while the torsioned ones are found to be lower than those in the aforementioned studies [11, 16].

\subsection{Water flux}

Figures $12,13,14$ and 15 present the water flux at different Reynolds number for torsioned and non-torsioned spacers at constant inlet salinity $\left(c_{0}\right)=400$ and 600 (mol/ $\mathrm{m}^{3}$ ). It is important to mention that a significant difference between the minimum values of torsioned and non-torsioned spacers were obtained from the simulation, consequently, different color legends had to be used. For all cases, low water flux is found at recirculation and tight regions due to the high $\mathrm{CP}$; for instance, low water flux is usually found near the filament strands. Torsioned and non-torsioned spacers have different recirculation regions which can be seen reflected in the water flux distribution on both membrane walls. It is clear that the water flux on membrane surfaces is enhanced and promoted with the unit cell containing the torsioned spacer, oppositely found with the non-torsioned spacer where water flux is significantly degraded along the spacer. It is clearly seen from Figs. $12,13,14$ and 15 the effect of increasing the inlet salinity from $\left(c_{\mathrm{o}}\right)=400$ to $600\left(\mathrm{~mol} / \mathrm{m}^{3}\right)$ on the water flux which is decreased substantially over the unit cell. On the other hand, it is noticed how enhanced the water flux is when increasing the Reynolds number from 100 to 384.5, especially, with locations containing high CP.

Figure 16a1, b1 describes the water flux at the upper membrane for non-torsioned and torsioned spacer at different inlet velocities with constant inlet salinity 

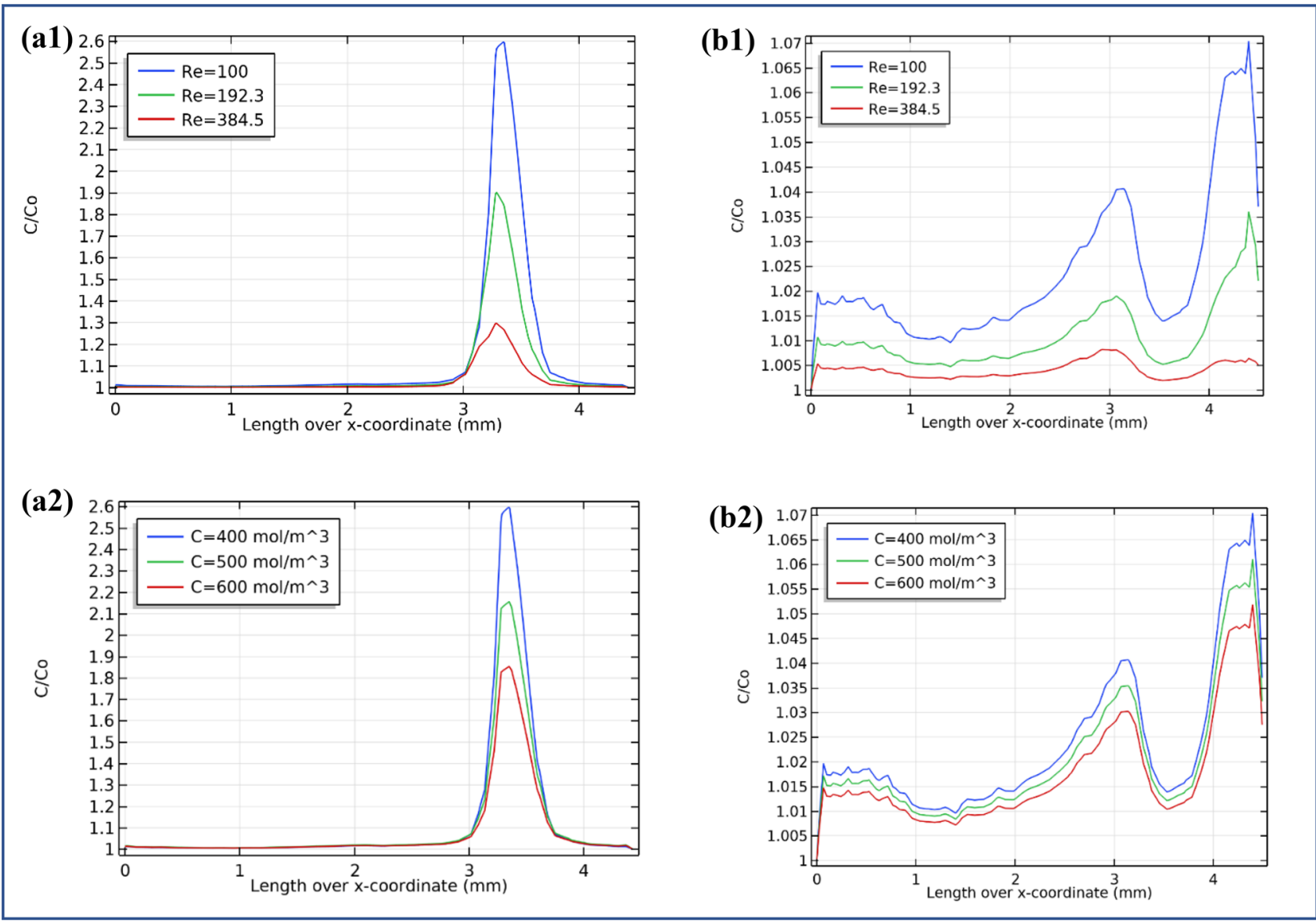

Fig. 11 Concentration profiles along the upper membrane surface a1, b1 for non-torsioned and torsioned spacers, respectively, at different Re and constant $c_{0}=400 \mathrm{~mol} / \mathrm{m}^{3}$ and $\mathbf{a 2}$, b2 for non-tor-

$\left(c_{0}\right)=400\left(\mathrm{~mol} / \mathrm{m}^{3}\right)$. Figure 16a2, b2 illustrates the effect of increasing the inlet salinity on the water flux at constant Reynolds number $(\operatorname{Re}=100)$ for both mentioned spacers at the upper membrane. It is obvious from the graphical representations that the torsioned spacer has a higher water flux rate compared to non-torsioned spacer. The highest water flux speed attained by the torsioned and non-torsioned spacer are $10.04 \times 10^{-6}(\mathrm{~m} / \mathrm{s})$ and $10 \times 10^{-6}(\mathrm{~m} / \mathrm{s})$, respectively at $\mathrm{Re}=384.5$ and inlet salinity $\left(c_{0}\right)=400\left(\mathrm{~mol} / \mathrm{m}^{3}\right)$, no significant effect is observed in means of the maximum water flux rate reached by both spacers geometry; however, the drawbacks come at high $\mathrm{CP}$ regions where a clear difference is witnessed between both spacers, for example, at $\operatorname{Re}=100$ and inlet salinity $\left(c_{0}\right)=600\left(\mathrm{~mol} / \mathrm{m}^{3}\right)$, the lowest water flux is seen with the non-torsioned spacer valued as $1.5 \times 10^{-6}(\mathrm{~m} / \mathrm{s})$, and with torsioned spacer valued as $7.2 \times 10^{-6}(\mathrm{~m} / \mathrm{s})$. Water flux is sioned and torsioned spacers, respectively, at different inlet salinity and constant $\mathrm{Re}=100$

found to be highly degraded with non-torsioned spacers compared to the torsioned ones. Furthermore, the graphs in Fig. 16 reveal that the torsioned spacer experienced two small falls located before and after the spacer, on the contrary with non torsioned spacers which experienced only one big fall located directly above the spacer, however in all cases, it is clear from the graphs that increasing the inlet salinity decreases the water flux drastically, and increasing Reynolds number increases the water flux. To sum up, the highest water flux is obtained at the lowest inlet salinity and at the highest Reynolds number, and the lowest water flux is obtained at the lowest Reynolds number and at the highest inlet salinity. Ma and Song [16] found the water flux to be $1.17 \times 10^{-5}(\mathrm{~m} / \mathrm{s})$ at $\mathrm{Re}=100$ and at inlet salinity $C_{o}=550\left(\mathrm{~mol} / \mathrm{m}^{3}\right)$. Also, Gu et al. [11] found the water flux between $6.1 \times 10^{-6}(\mathrm{~m} / \mathrm{s})$ and $7.3 \times 10^{-6}(\mathrm{~m} / \mathrm{s})$ at 


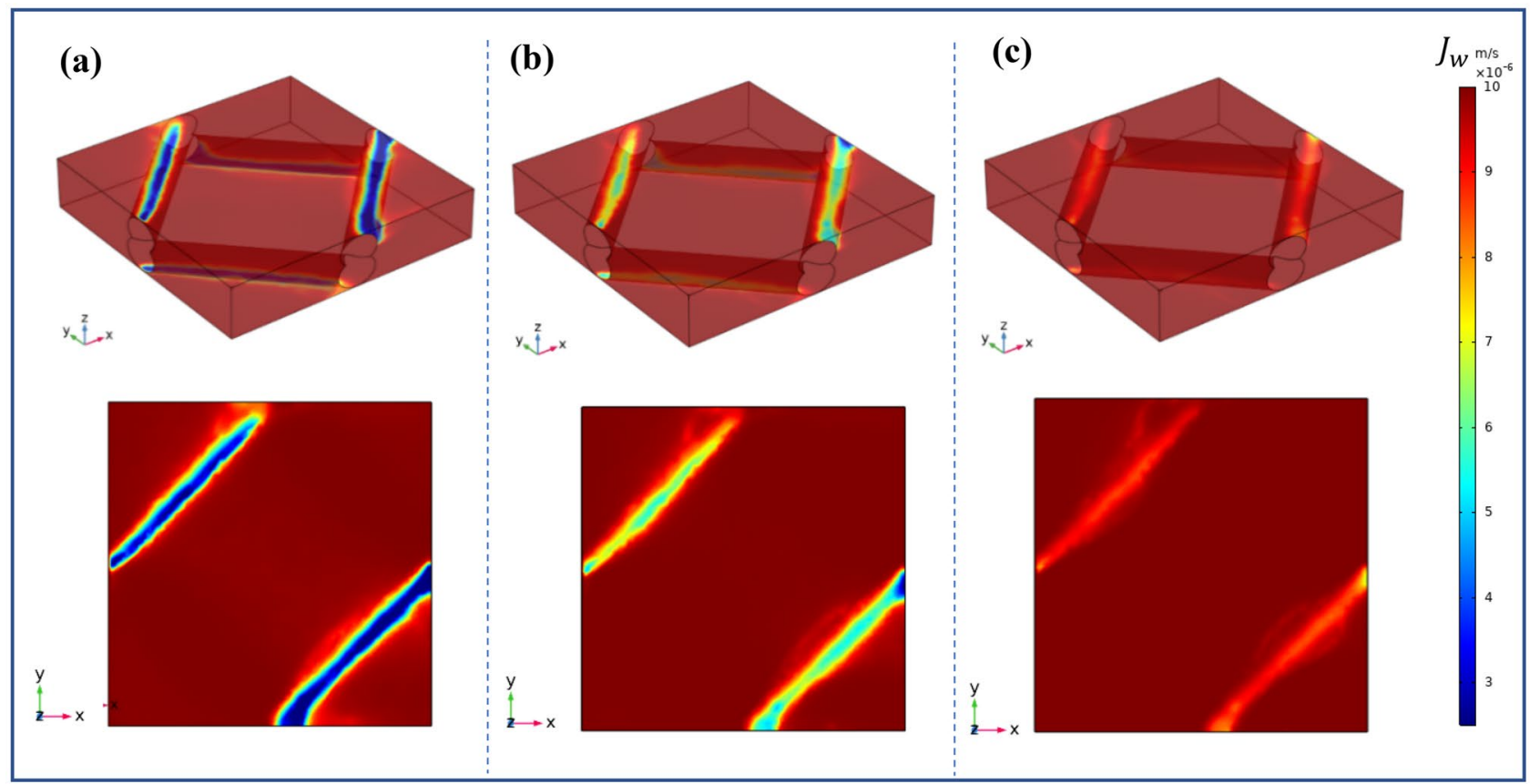

Fig. 12 Water flux of non-torsioned spacers at Re a 100, b 192.3 and c 384.5 with constant $c_{o}=400 \mathrm{~mol} / \mathrm{m}^{3}$ presented as isometric view and upper membrane surface

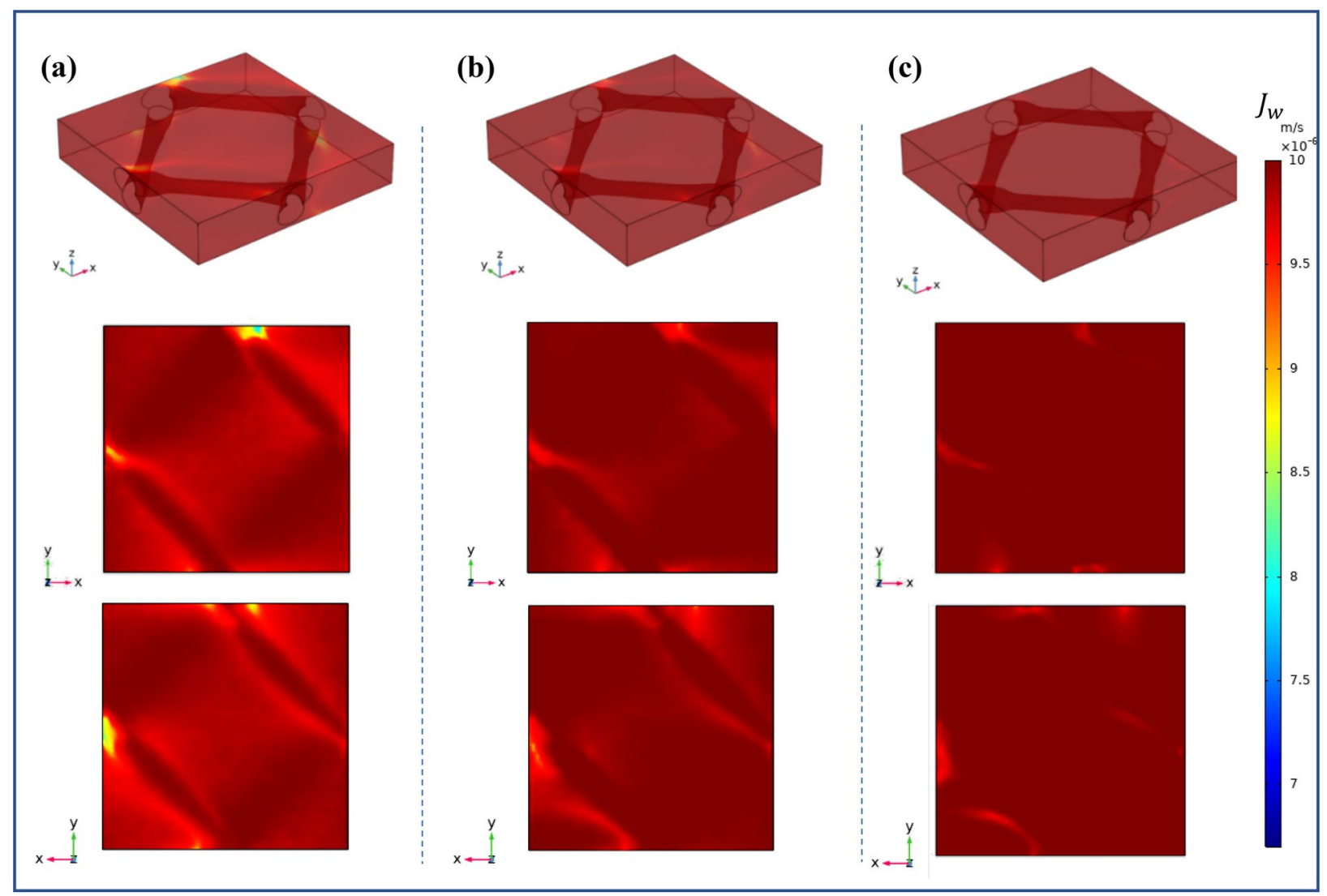

Fig. 13 Water flux of torsioned spacers at Re a 100, b 192.3 and c 384.5 with constant $c_{o}=400 \mathrm{~mol} / \mathrm{m}^{3}$ presented as isometric view, upper membrane surface and lower membrane surface

\section{SN Applied Sciences}




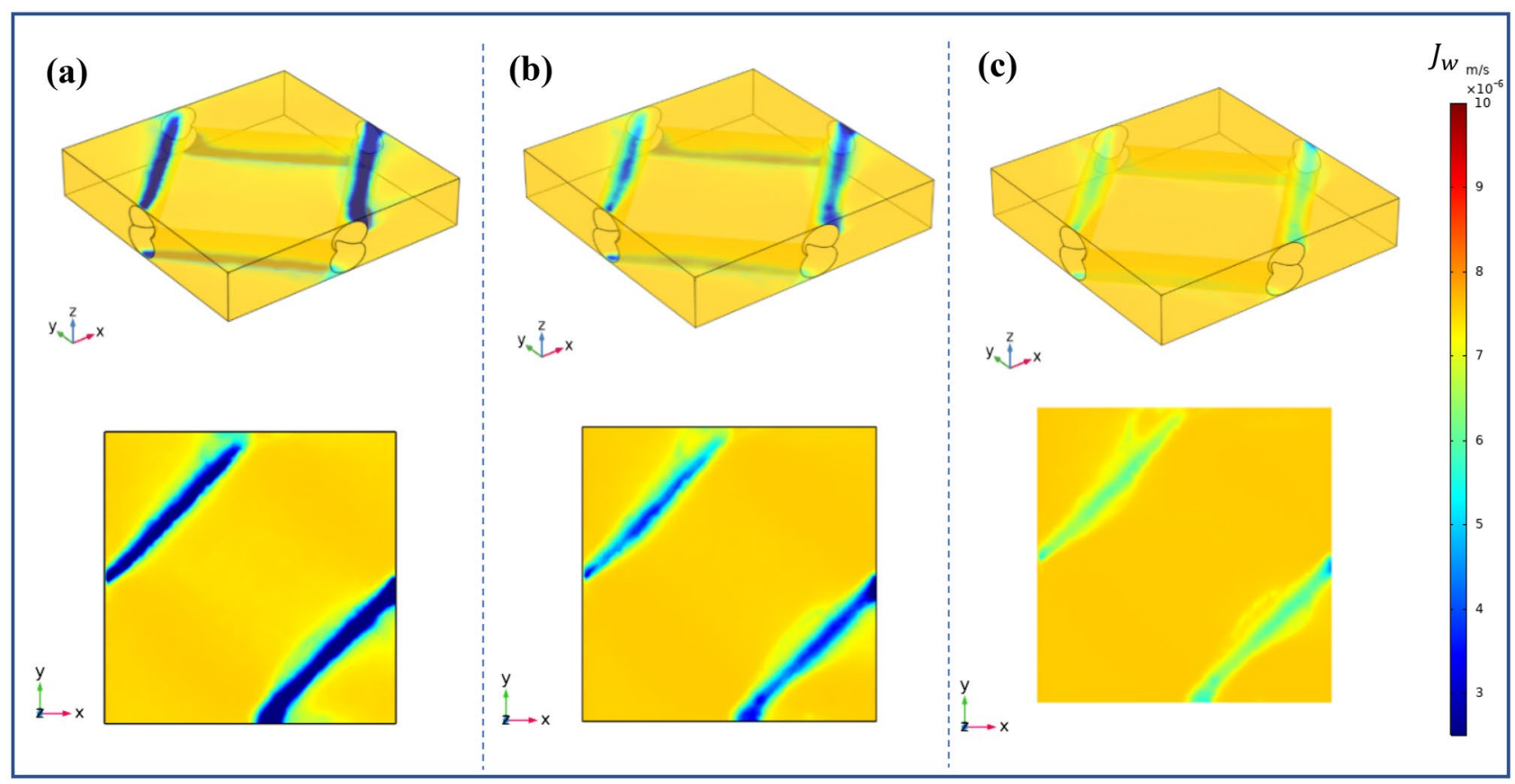

Fig. 14 Water flux of non-torsioned spacers at Re a 100 , b 192.3 and $\mathbf{c} 384.5$ with constant $c_{0}=600 \mathrm{~mol} / \mathrm{m}^{3}$ presented as isometric view and upper membrane surface

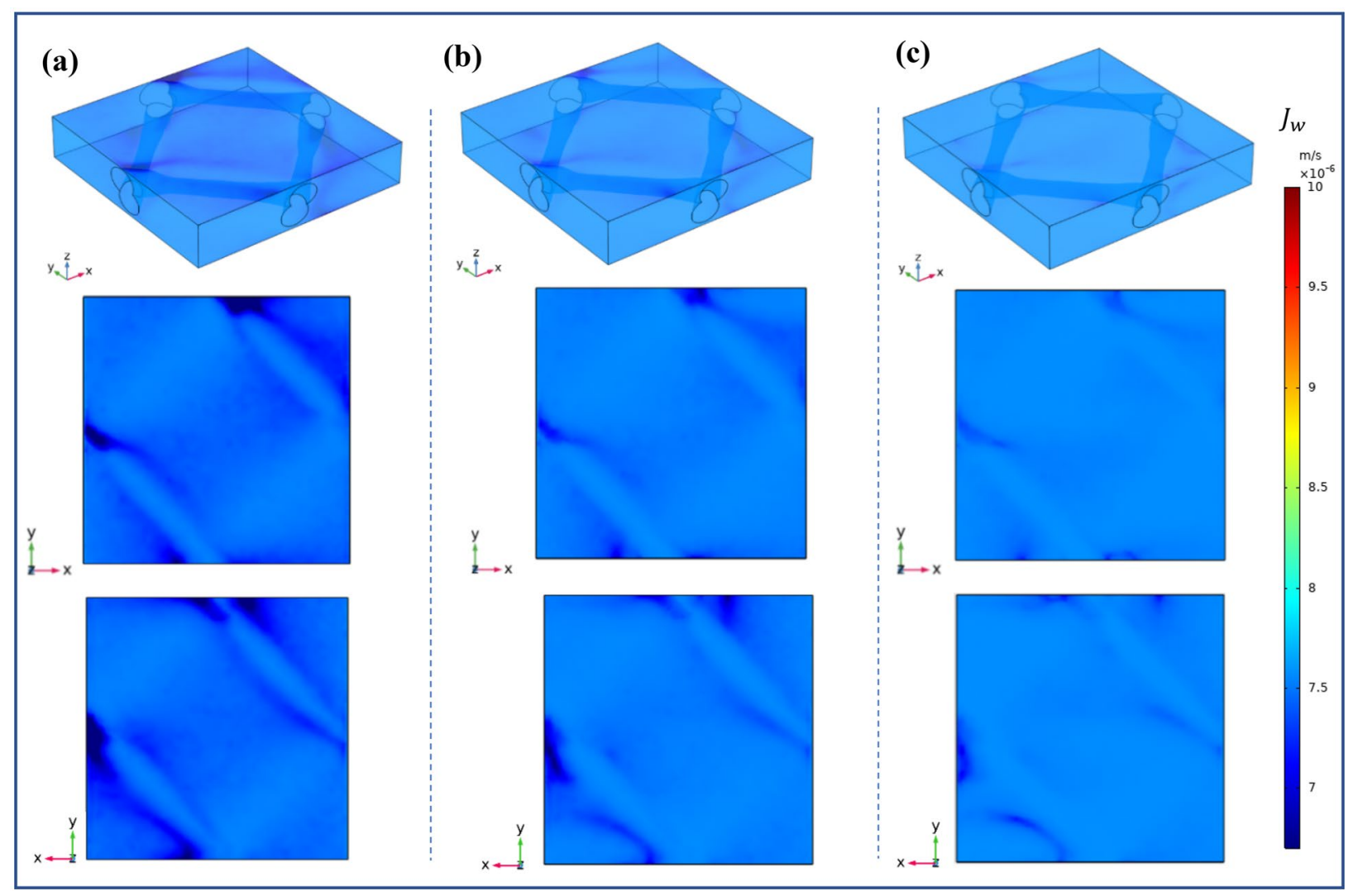

Fig. 15 Water flux of torsioned spacers at Re a 100, b 192.3 and c 384.5 with constant $c_{o}=600 \mathrm{~mol} / \mathrm{m}^{3}$ presented as isometric view, upper membrane surface and lower membrane surface 


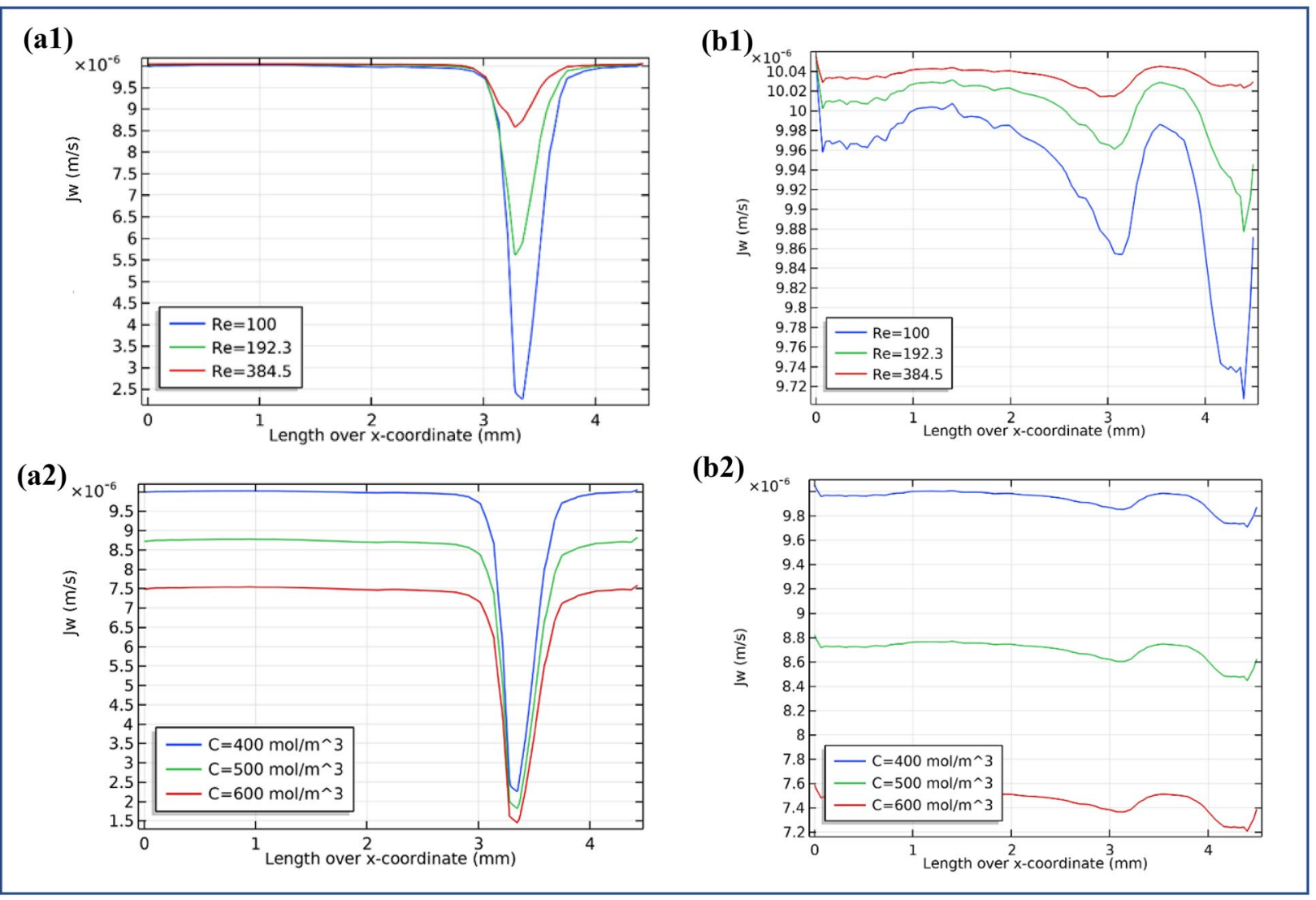

Fig. 16 Water flux along the upper membrane surface a1, b1 for non-torsioned and torsioned spacers, respectively, at different Re and constant $c_{o}=400 \mathrm{~mol} / \mathrm{m}^{3}$ and $\mathbf{a 2}, \mathbf{b} 2$ for non-torsioned and torsioned spacers, respectively, at different inlet salinity and constant $\mathrm{Re}=100$

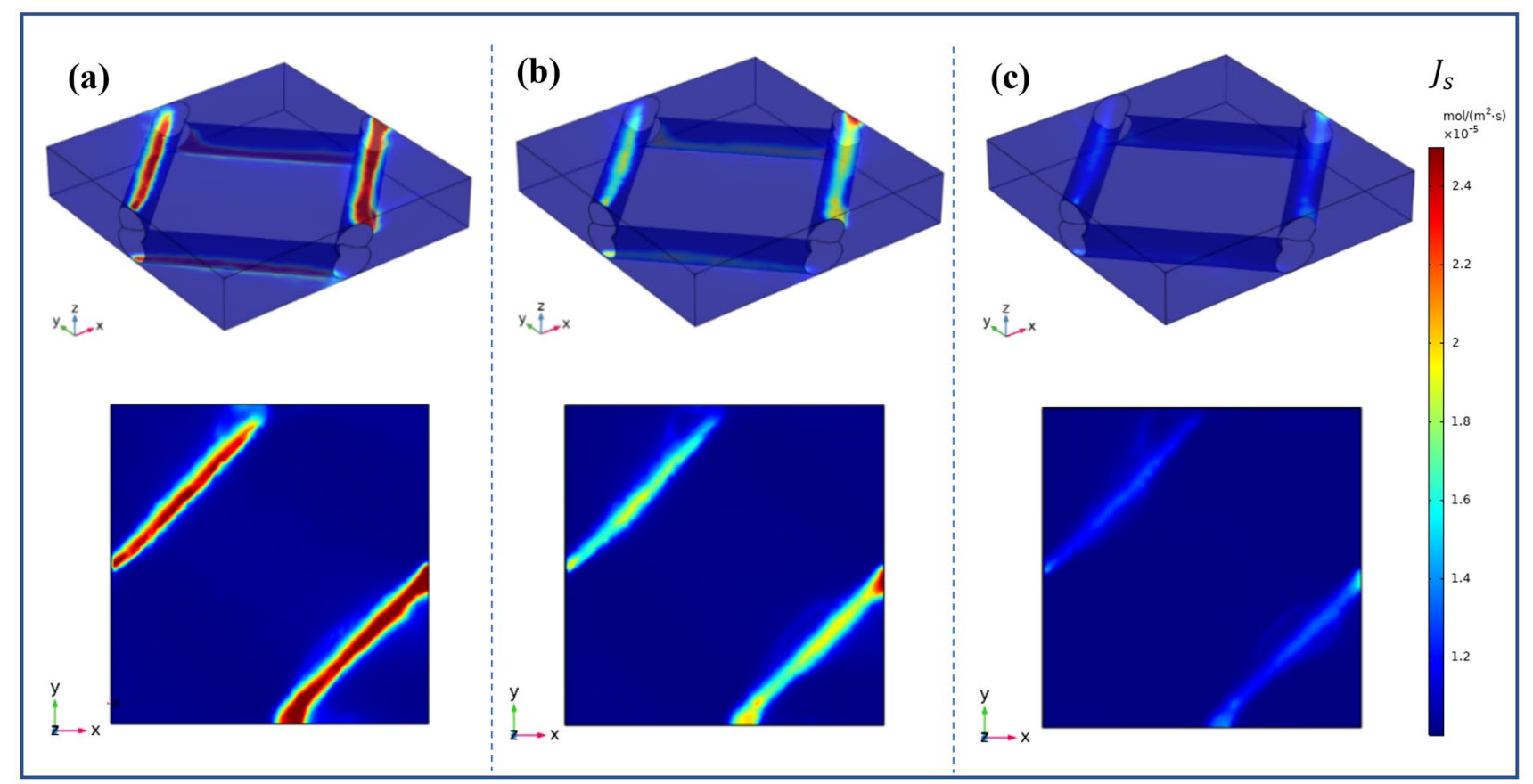

Fig. 17 Solute flux of non-torsioned spacers at Re a 100, b 192.3 and c 384.5 with constant $c_{o}=400 \mathrm{~mol} / \mathrm{m}^{3}$ presented as isometric view and upper membrane surface

\section{SN Applied Sciences}




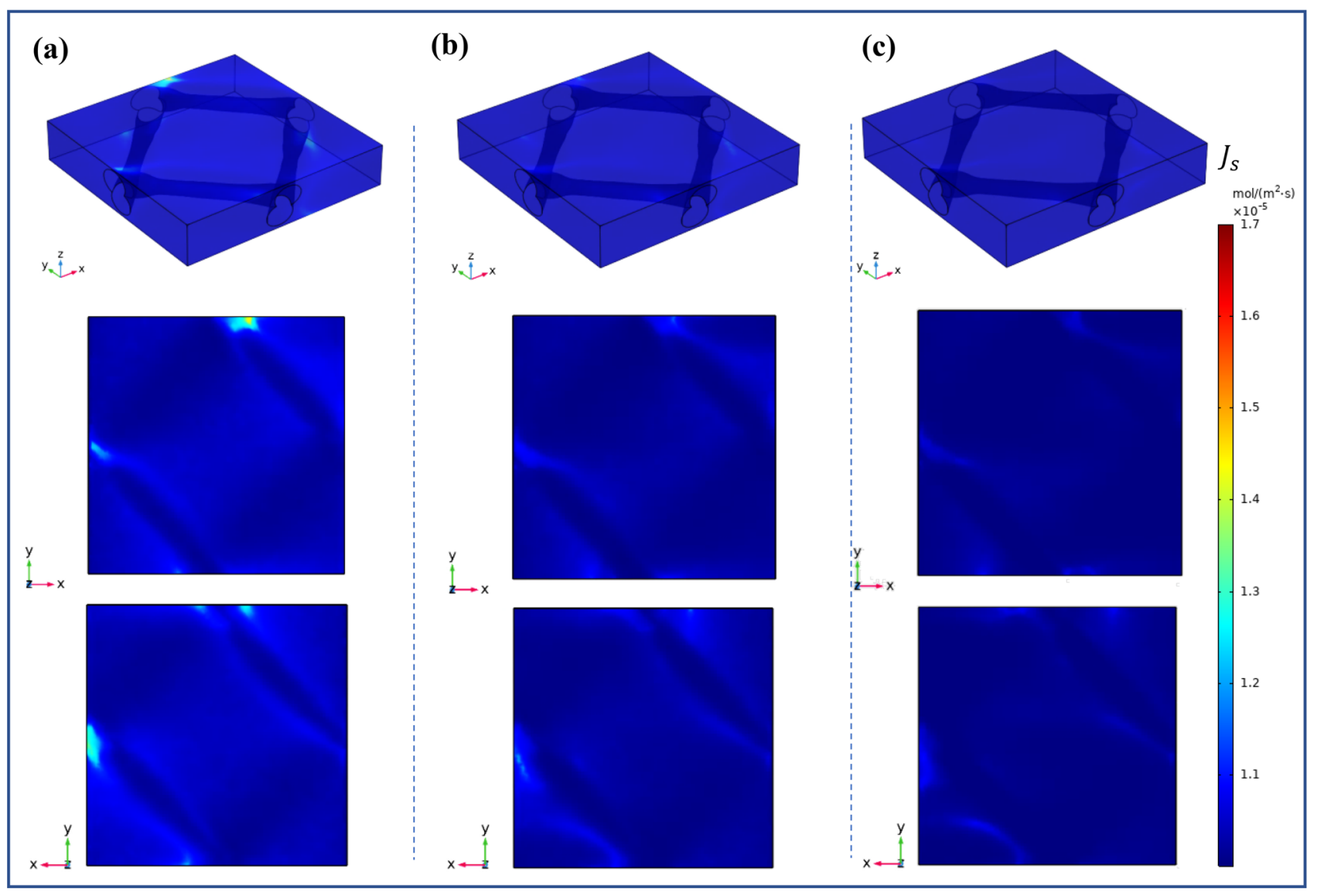

Fig. 18 Solute flux of torsioned spacers at Re a 100 , b 192.3 and c 384.5 with constant $c_{0}=400 \mathrm{~mol} / \mathrm{m}^{3}$ presented as isometric view, upper membrane surface and lower membrane surface

$\operatorname{Re}=224$ and inlet salinity $C_{o}=600\left(\mathrm{~mol} / \mathrm{m}^{3}\right)$. In the current study, the max water flux at $\operatorname{Re}=384.5$ and inlet salinity $\left(c_{0}\right)=400\left(\mathrm{~mol} / \mathrm{m}^{3}\right)$ is found to be $10.04 \times 10^{-6}(\mathrm{~m} / \mathrm{s})$ and $10 \times 10^{-6}(\mathrm{~m} / \mathrm{s})$ for torsioned and non-torsioned spacer, respectively.

\subsection{Solute flux}

Figures 17, 18, 19 and 20 depicts the distribution of solute flux at 3 different Reynolds number at constant inlet salinity $\left(c_{0}\right)=400$ and $600\left(\mathrm{~mol} / \mathrm{m}^{3}\right)$ for torsioned and nontorsioned spacers. The distribution of solute flux on membrane surfaces is similar to the $\mathrm{CP}$ where regions with high $\mathrm{CP}$ corresponds to regions with high solute flux agreeing with the formula of (Eq. 8) which states that the solute flux is proportional to the difference between the concentration of the feed and permeate. The solute flux tends to arise at the filament strands and at the gaps located between the spacers and membrane walls, it is an opposite reflection of the water flux. Similar to $\mathrm{CP}$ and water flux, solute flux distribution on membrane walls differ significantly between torsioned and non-torsioned spacers.
By observing the figures and taking into account the color legends, it is clear that the solute flux is higher and emphasized with the unit cell containing the non-torsioned spacer. It is noticed that with increasing the inlet salinity from $\left(c_{0}\right)=400$ to $600\left(\mathrm{~mol} / \mathrm{m}^{3}\right)$, the solute flux increases in the modeled unit cell, on the other hand by increasing Reynolds number from $R e=100$ to 384.5 , the solute flux decreases and mitigated especially with torsioned spacers.

Figure 21 is a graphical representation of the solute flux at different Reynolds number and at different inlet salinity. Figure 21a1, b1 illustrates the solute flux at the upper membrane for non-torsioned and torsioned spacer at $\operatorname{Re}=100,192.3$ and 384.5 with constant inlet salinity of $\left(c_{o}\right)=400\left(\mathrm{~mol} / \mathrm{m}^{3}\right)$. Figure $21 \mathrm{a} 2, \mathrm{~b} 2$ presents the effect of inlet salinity on the solute flux at constant Reynolds number $(R e=100)$ for both mentioned spacers at the upper membrane. At first glance, the graphs reveal that nontorsioned spacers have higher solute flux rates compared to torsioned spacers, e.g., the maximum solute flux in nontorsioned spacer is valued as $2.7 \times 10^{-5} \mathrm{~mol} / \mathrm{m}^{2} \mathrm{~s}$ and in the torsioned spacer as $1.57 \times 10^{-5} \mathrm{~mol} / \mathrm{m}^{2} \mathrm{~s}$ at $\mathrm{Re}=100$ and inlet salinity $\left(c_{0}\right)=600\left(\mathrm{~mol} / \mathrm{m}^{3}\right)$. The solute flux in 


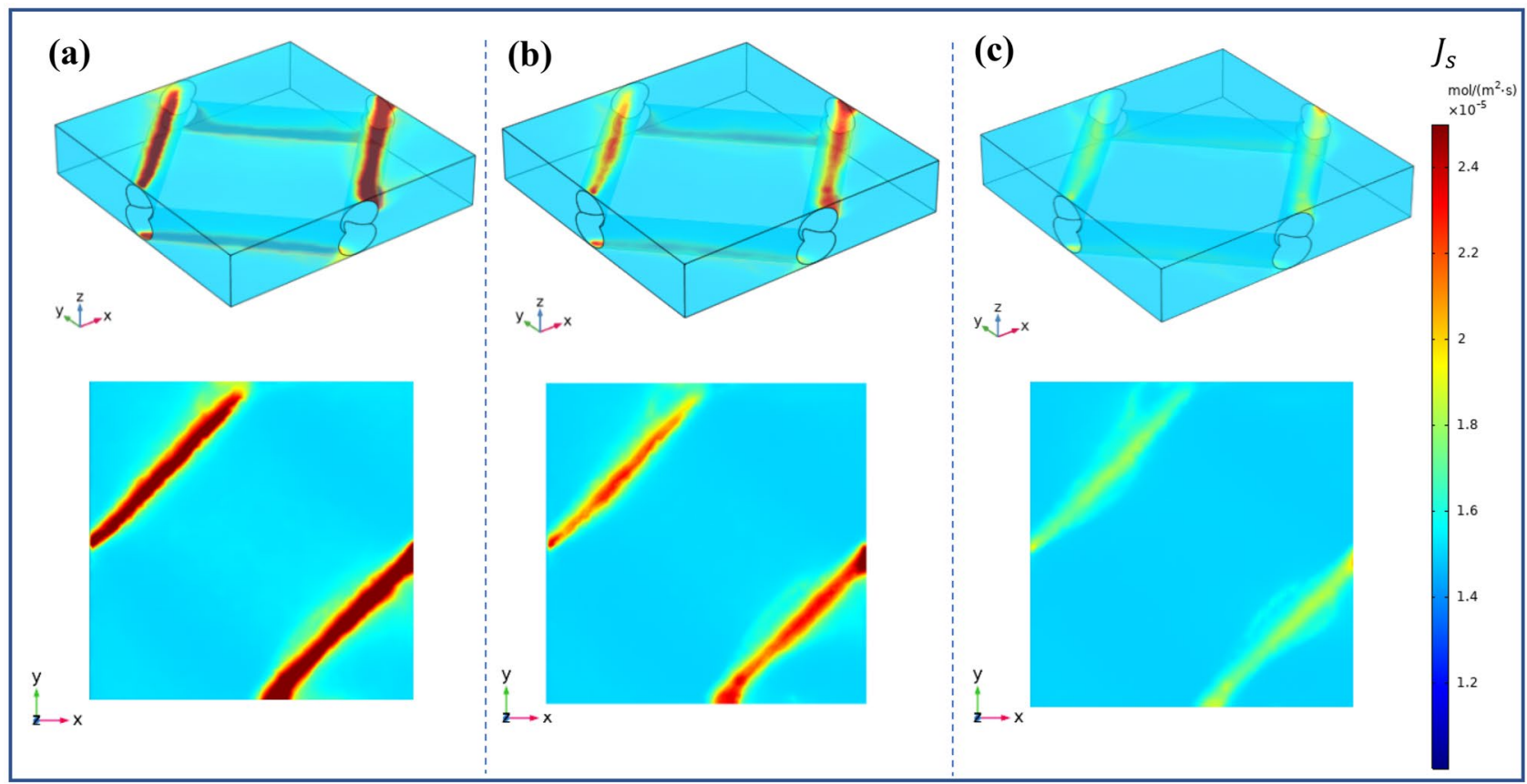

Fig. 19 Solute flux of non-torsioned spacers at Re a 100, b 192.3 and c 384.5 with constant $c_{o}=600 \mathrm{~mol} / \mathrm{m}^{3}$ presented as isometric view and upper membrane surface

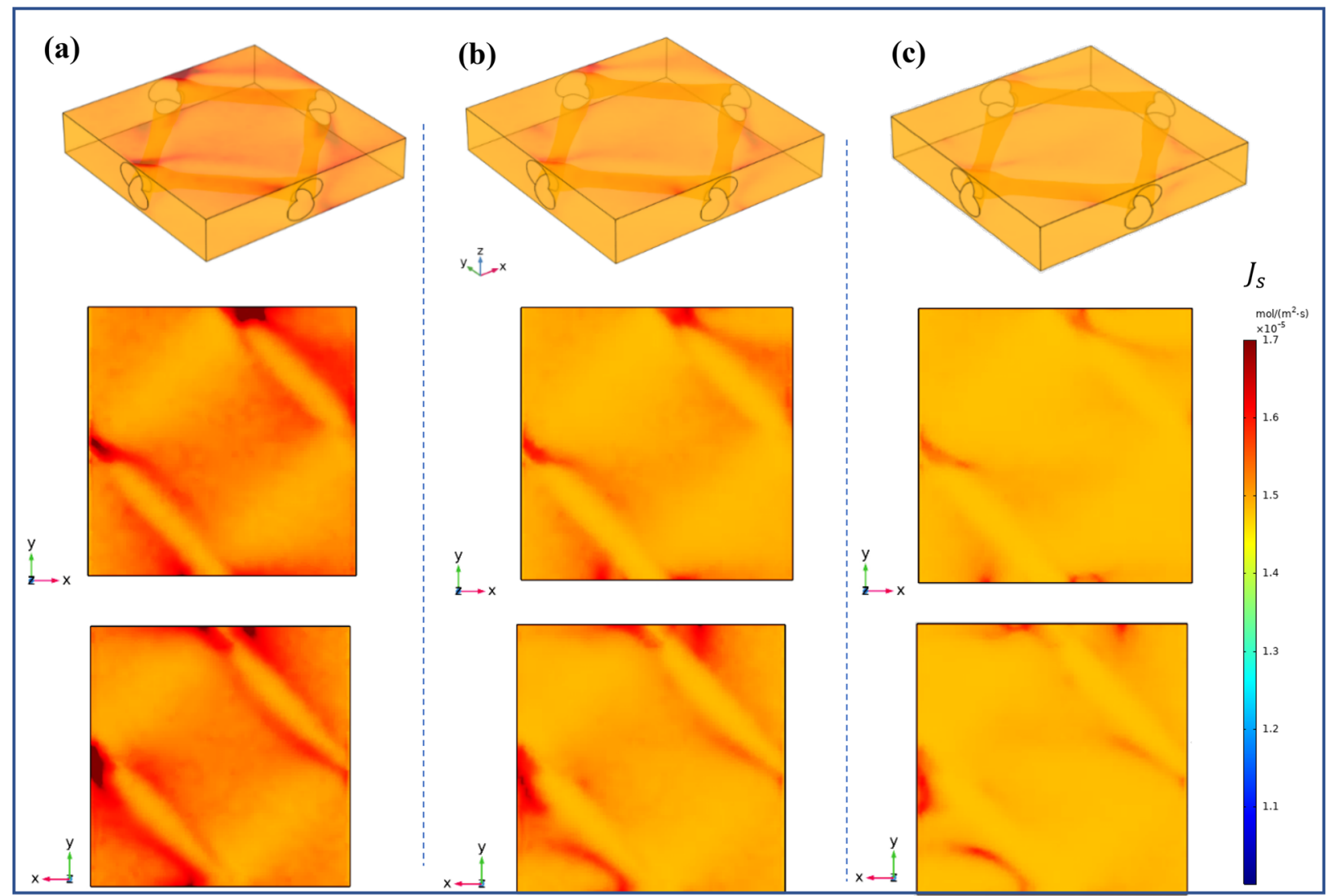

Fig. 20 Solute flux of torsioned spacers at Re a 100, b 192.3 and c 384.5 with constant $c_{o}=600 \mathrm{~mol} / \mathrm{m}^{3}$ presented as isometric view, upper membrane surface and lower membrane surface 




Fig. 21 Solute flux along the upper membrane surface a1, b1 for non-torsioned and torsioned spacers, respectively, at different Re and constant $c_{0}=400 \mathrm{~mol} / \mathrm{m}^{3}$ and $\mathbf{a 2} \mathbf{2}, \mathbf{b 2}$ for non-torsioned and torsioned spacers, respectively, at different inlet salinity and constant Re $=100$

Fig. 22 Comparison of pressure drop in torsioned and non-torsioned spacers in a unit cell

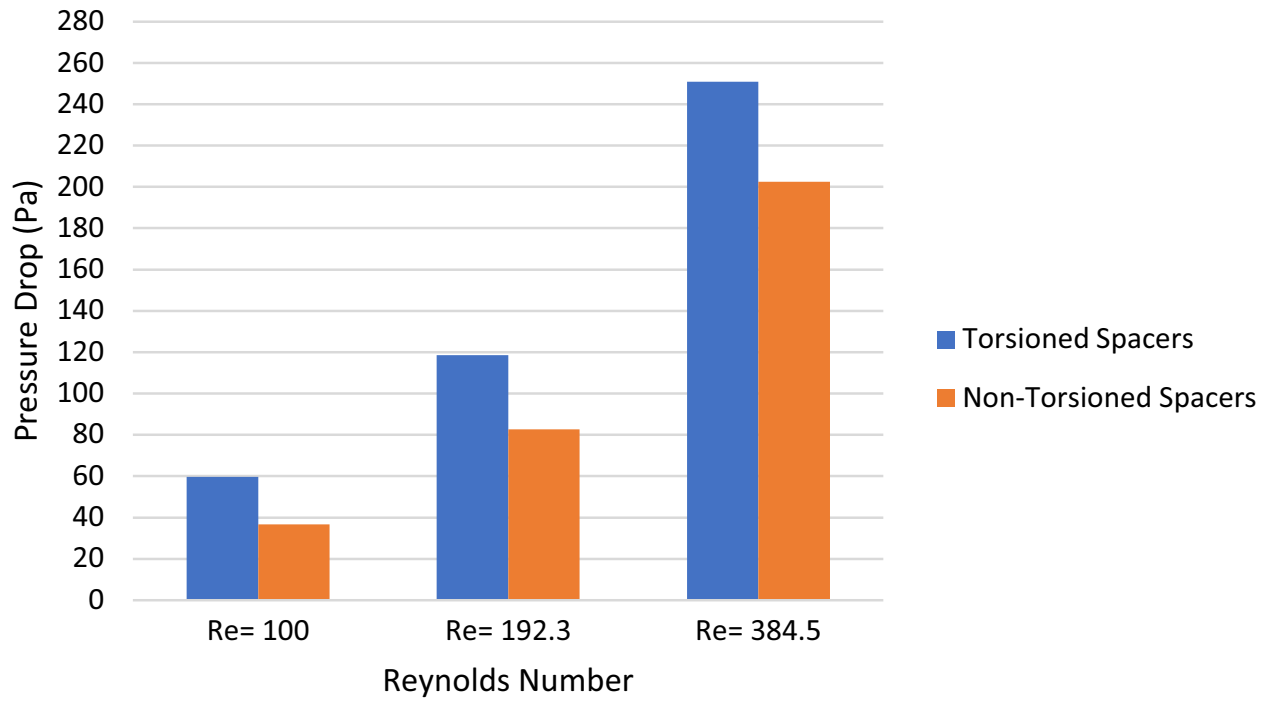

torsioned spacer is fluctuating along the membrane wall, on the contrary with torsioned spacer where the solute flux is seen to increase steadily along the membrane wall.
In all cases, as Reynolds number increases, the solute flux decreases, for instance, the maximum solute flux in nontorsioned spacer is equal to $2.57 \times 10^{-5}, 1.9 \times 10^{-5}$ and 
$1.3 \times 10^{-5} \mathrm{~mol} / \mathrm{m}^{2} \mathrm{~s}$ for $\mathrm{Re}=100,192.3$ and 384.5 at constant inlet salinity $\left(c_{0}\right)=400\left(\mathrm{~mol} / \mathrm{m}^{3}\right)$, respectively. Also increasing the inlet salinity will increase the solute flux, e.g., the maximum solute flux for torsioned spacer is equal to $1.06 \times 10^{-5}, 1.34 \times 10^{-5}$ and $1.57 \times 10^{-5} \mathrm{~mol} / \mathrm{m}^{2} \mathrm{~s}$ for inlet salinity $\left(c_{0}\right)=400,500$ and $600\left(\mathrm{~mol} / \mathrm{m}^{3}\right)$, respectively.

\subsection{Pressure drop}

The pressure drop in the current study was examined for both torsioned and non-torsioned spacers at different Reynolds number as presented in Fig. 22. It is obvious from the graph that the pressure drop is directly proportional to Reynolds number as was proven in previous studies [7, 17-19].

As it is illustrated in Fig. 22, the torsioned spacer has a higher pressure drop compared to the non-torsioned ones, for instance, at $R e=100$, the pressure drop is $59.6 \mathrm{~Pa}$ and 36.8 for the torsioned and non-torsioned spacers, respectively. This is due to the disturbance caused by the torsioned spacers because of the gap that is between the spacer and the membrane walls which is not found with non-torsioned spacer as it is attached along the membrane walls.

The spacer type has a significant effect on the pressure drop in spacer filled channels as it was elaborated by $\mathrm{Gu}$ et al. [11], they found the pressure drop at $R e=224$ to be between 8 and 70 Pa depending on the spacer type and mesh angle. The impact of increasing the inlet salinity on the pressure drop in the unit cell is seen to be negligible.

\section{Concluding remarks}

The current study adopted 18 different simulations performed on a 3D unit cell of a net-type non-woven torsioned and non-torsioned spacers for $\mathrm{RO}$ application with different inlet velocity and salinity. A comparison is presented between torsioned and regular spacers to investigate the effect of adopting such geometrical details in CFD studies, additionally, fluid dynamics and mass transfer are evaluated in different conditions. All simulations are solved using the fully coupled momentum and mass transport. One of the highlights of the current study is the use of realistic boundary conditions taken from Gu et al. [11] allowing accurate predictions of membrane performance. Based on the results obtained from the simulations, the following conclusions can be presented;

(a) Concentration polarization factor; is mitigated in torsioned spacers compared to non-torsioned spacers, $\mathrm{CP}$ is found in non-torsioned spacer to be symmetri- cal on the upper and lower membrane surfaces, on the contrary with torsioned spacer, where the $\mathrm{CP}$ is asymmetrical on the upper and lower membrane which is the result of the special effect induced by torsioned spacers. CP decreases with increasing inlet salinity and Reynolds number.

(b) Water flux; compared to non-torsioned spacers, is enhanced in torsioned spacers as CP is mitigated. The water flux is found decreasing as the inlet salinity increases and as Reynolds number decreases.

(c) Solute flux; similar to $\mathrm{CP}$, is lowered in torsioned spacers compared to non-torsioned spacers. Increasing the inlet salinity and decreasing the Reynolds number, increases the solute flux.

(d) Pressure drop; is higher in torsioned spacers compared to non-torsioned spacers.

To sum up, the torsioned spacers outperform the nontorsioned spacers proving the importance of the geometrical details of feed spacers, and for all cases, increasing the inlet salinity and decreasing Reynolds number, degrades the performance of RO membrane.

Acknowledgements The author gratefully acknowledges the support of Prof. Boram Gu and Prof. Xia Yun Xu from the imperial college London who made this research possible.

\section{Compliance with ethical standards}

Conflict of interest On behalf of all authors, the corresponding author states that there is no conflict of interest.

\section{References}

1. Shakaib M, Hasani SM, Mahmood M (2007) Study on the effects of spacer geometry in membrane feed channels using three-dimensional computational flow modeling. J Membr Sci 297(1-2):16

2. Saeed A, Vuthaluru R, Vuthaluru HB (2015) Impact of feed spacer filament spacing on mass transport and fouling propensities of RO membrane surfaces. Chem Eng Commun 202(5):634-646

3. Koutsou CP, Karabelas AJ (2012) Shear stresses and mass transfer at the base of a stirred filtration cell and corresponding conditions in narrow channels with spacers. J Membr Sci 399-400:13

4. Fimbres-Weihs GA, Wiley DE (2010) Review of 3d CFD modeling flow and mass transfer in narrow spacer-filled channels in membrane modules. Chem Eng Process 49:759-781

5. Cao Z, Wiley DE (2001) Fane AG CFD simulations of net-type turbulence promoters in a narrow channel. J Membr Sci 185:157-176

6. Karode SK, Kumar A (2001) Flow visualization through spacer filled channels by computational fluid dynamics I: Pressure drop and shear rate calculations for flat sheet geometry. J Membr Sci 193:69 
7. Koutsou CP, Yiantsios SG, Karabelas AJ (2007) Direct numerical simulation of flow in spacer-filled channels: effect of spacer geometrical characteristics. J Membr Sci 291(1-2):53-69

8. Fimbres-Weihs GA, Wiley DE (2007) Numerical study of mass transfer in three-dimensional spacer-filled narrow channels with steady flow. J Membr Sci 306(1-2):228-243

9. Ranade VV, Kumar A (2006) Fluid dynamics of spacer filled rectangular and curvilinear channels. J Membr Sci 271(1-2):1-15

10. Srivathsan $G$ (2014) Modeling of fluid flow in spiral wound reverse osmosis membranes. University of Minnesota, Minnesota

11. Gu B, Adjiman CS, Xu XY (2017) The effect of feed spacer geometry on membrane performance and concentration polarisation based on 3D CFD simulations. J Membr Sci 527:78-91

12. Anqi AE, Alkhamis N, Oztekin A (2016) Computational study of desalination by reverse osmosis-Three-dimensional analyses. Desalination 388:38-49

13. Xie P (2016) Simulation of reverse osmosis and osmotically driven membrane processes. Graduate School of Clemson University, Clemson, $\mathrm{p} 112$

14. Shakaib M, Hasani SMF, Mahmood M (2009) CFD modeling for flow and mass transfer in spacer-obstructed membrane feed channels. J Membr Sci 326(2):270-284

15. Haidari AH, Heijman SGJ, van der Meer WGJ (2018) Effect of spacer configuration on hydraulic conditions using PIV. Sep Purifi Technol 199:9-19
16. Song L, Ma S (2005) Numerical studies of the impact of spacer geometry on concentration polarization in spiral wound membrane modules. Ind Eng Chem Res 44(20):7638-7645

17. Schock G, Miquel A (1987) Mass transfer and pressure loss in spiral wound modules. Desalination 64:339-352

18. Koutsou C, Karabelas A, Kostoglou M (2018) Fluid dynamics and mass transfer in spacer-filled membrane channels: effect of uniform channel-gap reduction due to fouling. Fluids 3(1):12

19. Anqi AE, Alkhamis N, Oztekin A (2015) Numerical simulation of brackish water desalination by a reverse osmosis membrane. Desalination 369:156-164

20. Haidari AH, Heijman SGJ, van der Meer WGJ (2016) Visualization of hydraulic conditions inside the feed channel of reverse osmosis: a practical comparison of velocity between empty and spacer-filled channel. Water Res 106:232-241

Publisher's Note Springer Nature remains neutral with regard to jurisdictional claims in published maps and institutional affiliations. 